\title{
Éléments pour un institutionnalisme méthodologique : autonomie, variation d'échelle, réflexivité et abduction
}

Agnès Labrousse

\section{(2) OpenEdition}

Journals

Édition électronique

URL : http://journals.openedition.org/ei/1107

DOI : 10.4000/ei. 7107

ISSN : 2553-1891

Éditeur

Association Économie et Institutions

Édition imprimée

Date de publication : 1 juin 2006

Pagination : 5-53

ISSN : 1775-2329

Référence électronique

Agnès Labrousse, «Éléments pour un institutionnalisme méthodologique : autonomie, variation d'échelle, réflexivité et abduction », Économie et institutions [En ligne], 8 | 2006, mis en ligne le 31 janvier 2013, consulté le 10 décembre 2020. URL : http://journals.openedition.org/ei/1107 ; DOI : https:// doi.org/10.4000/ei.1107 
Eléments pour un institutionnalisme méthodologique :
autonomie, variation d'échelle, réflexivité et abduction*

\section{Agnès Labrousse ${ }^{1}$,}

\section{Introduction ${ }^{2}$}

"La réalité économique ne porte jamais sur des corps simples ". Fernand Braudel (1976, p. 54)

Les systèmes économiques sont constitués d'éléments multiples. A leur base, on trouve des acteurs individuels et collectifs. Ces myriades d'acteurs prennent des décisions économiques à chaque instant. Comment analyser ces décisions et leurs produits sachant que les motivations de ces acteurs ne sont pas déterminées a priori par quelque grand organisateur ou ordonnateur du monde, c'est-à-dire sachant que ces acteurs bénéficient d'une relative autonomie par rapport à leur environnement ?

Depuis l'autonomia des cités grecques, la notion d'autonomie, dans ses applications à des domaines aussi divers que la psychologie de l'enfant ou les mouvements syndicaux, renvoie toujours à l'idée d'une entité qui se régit par ses propres lois. Pour être autonome, un ensemble doit disposer de frontières propres et se distinguer par là de l'environnement qui l'entoure. En même temps, il ne doit pas être complètement imperméable aux influences extérieures. Car l'autonomie trouve son origine dans un certain degré d'ouverture/fermeture à l'égard de l'environnement dont l'action va être filtrée par des processus internes. "Un système absolument fermé serait une boîte noire dont on ne peut rien dire puisque rien

* L'auteur tient à remercier chaleureusement Stéphane Longuet et les participants du séminaire ORIS du CRIISEA ainsi que Catherine ColliotThélène et les trois rapporteurs anonymes de ce texte pour leurs très précieuses critiques. Afin de ne pas étoffer à l'excès un texte déjà long, il n'a pas toujours été possible de les intégrer complètement. Les erreurs et manques qui ne manqueront pas de subsister sont bien entendu de notre entière responsabilité.

1 CRIISEA, Université de Picardie - Jules Verne

2 Ce texte trouve son origine dans une invitation de Stéphane Longuet à réfléchir sur la notion d'autonomie et ses implications en économie dans le cadre du séminaire ORIS. Cela éclaire pour partie la place accordée à cette notion dans le présent article, une place peu usuelle dans la littérature méthodologique existante. Cette "figure imposée" par la thématique du séminaire, si elle peut parfois prendre un tour artificiel, s'est avérée présenter un intérêt heuristique important au fur et à mesure que nous progressions. 
n'y entrerait et rien n'en sortirait. Un système absolument ouvert serait sans frontière, indiscernable de son environnement " (Lapierre, 1992, p. 27). En cela, l'autonomie s'oppose à l'hétéronomie où les lois sont définies de l'extérieur (Weber, 1922) mais aussi à l'autisme où l'environnement paraît absent, étranger. L'autiste n'est-il pas replié, fermé sur lui-même, en un monde clos?

La conceptualisation de l'autonomie fait généralement appel aux théories des systèmes complexes (Prigogine \& Stengers, Varela, Atlan, Simon, Morin, Le Moigne etc.). Celles-ci fournissent un métalangage scientifique demandant à être traduit dans chaque discipline. En économie, le terrain de la complexité n'est que partiellement balisé par deux prix Nobel : Friedrich Hayek et Herbert Simon. On en trouve également des échos dans les approches régulationnistes, notamment chez Robert Delorme, ou encore dans l'analyse comparative des systèmes de Janos Kornai.

Dans cette perspective, ce texte propose de prendre pour point de départ la notion d'autonomie pour cheminer progressivement vers l'institutionnalisme méthodologique. Trois déclinaisons de la notion d'autonomie seront examinées : l'autonomie des niveaux d'observation, l'autonomie de l'observateur par rapport au réel, l'autonomie des acteurs. Sur cette base, divers principes méthodologiques peuvent être dégagés. Ces principes peuvent être aisément intégrés au sein des théories relevant de l'institutionnalisme historique ${ }^{3}$ alors même que la théorie standard ${ }^{4}$ s'avère, telle est en tout cas notre thèse, largement incompatible avec la prise en compte de l'autonomie. L'institutionnalisme historique est représenté par des courants aussi divers que l'école historique allemande (Schmoller, Weber) ${ }^{5}$, l'institutionnalisme américain de Commons et Veblen, l'ordolibéralisme allemand (Eucken) ou encore la théorie française de la régulation ${ }^{6}$. Il va de soi que les positions de

3 Sur les différents types d'institutionnalisme et la notion d'institutionnalisme historique, on se reportera à Thelen \& Steinmo (1992) et à Théret (2000).

4 Nous faisons ici référence à la distinction opérée par Olivier Favereau (1989) entre théorie standard et théorie standard élargie.

5 Nous incluons résolument l'école historique allemande dans ce panorama. En raison de préjugés tenaces et d'une méconnaissance de ses productions, elle occupe à tort une place minime dans le renouveau institutionnaliste actuel : Schmoller sert encore de repoussoir à nombre d'analyses et Weber est confiné hors du champ de l'économie.

6 Il n'est pas possible de traiter ici du débat sur les frontières entre approches institutionnalistes. Il nous semble cependant que l'institutionnalisme historique, contrairement à d'autres courants institutionnalistes, a pour noyau dur une prise en compte de la spécificité historique des systèmes économiques. Ces courants ont donc pour visée la mise en évidence de régularités situées dans le temps et dans l'espace et non

6 Economie et Institutions $-\mathrm{n}^{\circ} 8-1^{\mathrm{er}}$ semestre 2006 
ces courants et de ces auteurs sont loin d'être identiques. Nous laissons ici sciemment dans l'ombre ces variations pour nous concentrer sur leurs caractéristiques communes, centrales pour notre propos. Ce texte se veut une contribution, nécessairement modeste et partielle, à un "institutionnalisme méthodologique " qui dispose de soubassements déjà anciens mais demeure fragmentaire. Il espère, en ne restant pas uniquement à un niveau métathéorique, apporter, autant que faire se peut, quelques propositions méthodologiques concrètes. Celles-ci prennent fréquemment appui sur des méthodologies développées dans d'autres sciences sociales, de la sociologie à l'histoire en passant par l'ethnologie. Cette réflexion participe du mouvement constructiviste et, plus spécifiquement du pragmatist turn, du linguistic turn, et du cognitive turn en sciences sociales. Un mouvement certes protéiforme et parfois contradictoire mais qui a sans doute trouvé trop peu d'échos au sein de la discipline économique ${ }^{7}$.

Dans une première partie, l'examen des trois dimensions de l'autonomie évoquées plus haut permettra de mettre en évidence les problèmes d'échelle d'observation, la nécessité d'une approche réflexive et d'une démarche d'enquête ouverte. Dans la seconde, le lien entre autonomie et institutionnalisme est exploré plus avant afin de dégager les principaux éléments d'une analyse institutionnaliste située historicisant à la fois l'observateur et son objet. Le chercheur y obéit à une rationalité procédurale suivant une logique abductive où l'observation, la nouveauté et la surprise jouent un rôle moteur dans le processus de construction théorique.

\section{DE LA NOTION D'AUTONOMIE A L'INSTITUTIONNALISME METHODOLOGIQUE}

Nous venons d'évoquer brièvement l'autonomie des acteurs économiques dans les processus de prise de décision, une autonomie qui a, nous allons le voir, de nombreuses implications pour le chercheur. Mais ce dernier constitue lui-même un acteur spécifique, qui développe un regard propre sur son objet d'étude. Cette autonomie du chercheur mérite également d'être examinée. Les décisions des acteurs et leurs conséquences peuvent en outre être perçues et analysées à différents niveaux (du micro au macro). Comme nous le verrons, on n'observe pas les mêmes phénomènes

de lois universelles. Sur la notion de spécificité historique, on se reportera à Hodgson (2001) et au point 2.2. du présent texte.

7 Sur le caractère protéiforme du pragmatisme en philosophie, $c f$. Laugier (1999). Sur la diversité des courants constructivistes, on se reportera aux travaux de Hacking (2001) ; cf. également Orléan A. (2002). 
selon l'échelle adoptée ; chaque niveau d'observation est doté d'une certaine autonomie par rapport aux autres niveaux.

\subsection{Autonomie et échelles d'observation}

"Les systèmes économiques réels sont des systèmes multi-niveaux ". Janos Kornai [1971]

\section{Autonomie et niveaux d'intégration}

Les systèmes économiques, comme tous les systèmes complexes, sont composés de différents niveaux d'intégration emboîtés les uns dans les autres. "Un système est un objet complexe, formé de composants distincts reliés entre eux par un certain nombre de relations. Les composants sont considérés comme des sous-systèmes, ce qui signifie qu'ils entrent dans la même catégorie d'entités que les ensembles auxquels ils appartiennent. Un sous-système peut être décomposé à son tour en sous-systèmes d'ordre inférieur ou être traité (au moins provisoirement) comme un système indécomposable, c'est-à-dire comme un système réduit à un seul élément. L'idée essentielle est que le système possède un degré de complexité plus grand que ses parties, autrement dit qu'il possède des propriétés irréductibles à celles de ses composants " (Ladrière, 1995). En économie, on peut classiquement distinguer entre les niveaux micro-, méso- et macroéconomique ${ }^{8}$. Ces niveaux sont emboîtés hiérarchiquement avec des phénomènes de rétroaction ( $c f$. figure 1).

Ainsi, le niveau microéconomique des entreprises et des ménages est intégré dans les niveaux d'agrégation supérieurs (sectoriel, national, international). L'organisation-entreprise est contrainte par des règles et régularités nationales et sectorielles qui viennent conditionner son comportement et sa viabilité sans pour autant le déterminer. Ses frontières, à l'image de parois osmotiques, sont poreuses (ouverture à l'environnement) et mouvantes (mouvements d'externalisation et d'internalisation). L'autonomie de chaque niveau résulte d'un filtrage interne des influences exercées par les niveaux d'intégration supérieurs. "Les pressions de sélection ne s'exercent pas sur un système qui serait neutre par rapport à elles : c'est le comportement du système, conséquence de son état antérieur d'organisation et de la ou des mutations qui s'y sont produites, qui oriente les pressions de sélection dans une direction donnée plutôt qu'une autre" (Atlan [1972], p. 58). Les acteurs

8 On peut définir une gradation du plus micro (niveau individuel) au plus macro (économie mondiale) en passant par une infinité de niveaux intermédiaires.

8 Economie et Institutions $-n^{\circ} 8-1^{\text {er }}$ semestre 2006 
individuels, les organisations, les systèmes sectoriels, les systèmes économiques nationaux sont porteurs de mémoires propres, sélectives et agissantes dans le présent. Des mémoires qui définissent des répertoires de ressources, des modes de réaction aux changements de l'environnement. Aussi les conditions initiales et les expériences passées contribuent à la définition des trajectoires à chaque niveau.

Pour comprendre l'autonomie relative de chaque niveau, la notion d'émergence est fondamentale. Elle renvoie à l'idée que de nouvelles propriétés émergent dans un système complexe qui ne sont pas réductibles à ses éléments constitutifs de niveau inférieur. Le biologiste Ernst Mayr (cité in Hodgson, 1998, p. 157) la définit ainsi : " les systèmes, à chaque niveau hiérarchique, ont une double caractéristique. Ils agissent comme des tout (wholes) et leurs caractéristiques ne peuvent (même pas en théorie) être déduites de la connaissance la plus complète de leurs composants, qu'ils soient pris séparément ou en combinaisons partielles. En d'autres termes, lorsque ces systèmes sont assemblés à partir de leurs composants, de nouvelles caractéristiques de ce nouveau tout émergent qu'il n'était pas possible de prédire à partir d'une connaissance des composants. La reconnaissance de l'importance de l'émergence démontre, bien entendu, l'invalidité d'un réductionnisme extrême. Au moment où nous avons disséqué un organisme jusqu'à ses atomes et particules élémentaires, nous avons perdu tout ce qui est caractéristique d'un système vivant".

Figure 1: Un exemple d'interrelations entre trois niveaux d'intégration économiques

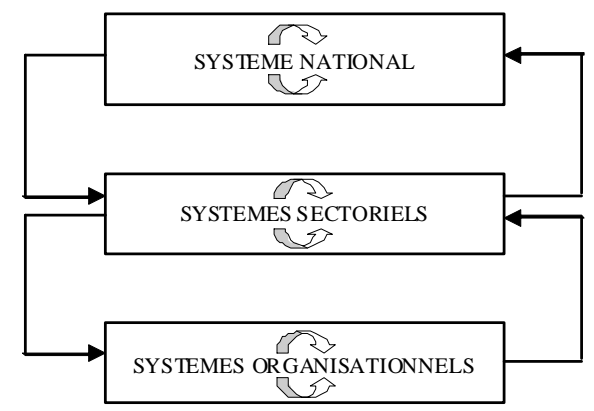

LEGENDE

$\downarrow \quad$ Cont rainte de conformité, de compatibilité du niveau inférieur par rapport au niveau supérieur

$\uparrow$ Rétroactions du niveau inférieur vers le niveau supérieur 
Il s'agit donc de dénaturaliser - ou tout au moins, de débanaliser les mécanismes d'agrégation et d'association en insistant sur les modalités relationnelles qui les rendent possibles, en repérant les médiations entre rationalités individuelles et collectives. A chaque échelle d'observation, les phénomènes économiques apparaissent sous un jour différent. Une telle conclusion entre en contradiction avec les options méthodologiques de la théorie économique standard. En effet, dans le corpus standard, il n'y a pas d'échelle, de focale d'observation. Toute totalité étant décomposable en éléments individuels, le choix de l'échelle d'observation n'a aucune influence sur la nature des processus causaux efficients. Le réel est fondamentalement plat, unidimensionnel sans phénomènes d'émergence entre niveaux. Dans une vision systémique au contraire, le réel est rugueux, multidimensionnel, fractal. Comme le dit Kornai, les systèmes économiques sont des systèmes multi-niveaux.

On n'observe pas les mêmes phénomènes à chaque échelle

"Une ville, une campagne, de loin c'est une ville et une campagne, mais à mesure qu'on s'approche, ce sont des maisons, des arbres, des tuiles, des feuilles, des herbes, des fourmis, des jambes de fourmis, à l'infini. Tout cela s'enveloppe sous le nom de campagne ".

Pascal [1670], p. 61

"Le déroulement de l'analyse peut être comparé à un vol. A forte altitude, on peut souvent embrasser du regard tout un pays. Lorsque, ensuite, on se rapproche de la terre, on perçoit le réseau qui relie les différents points, les voies, les lignes de chemin de fer, les circuits interurbains etc. A distance moindre encore, on peut différencier des unités toujours plus petites comme les villes, des blocs de maisons, des maisons etc. Ensuite, l'avion reprend de l'altitude et l'on peut à nouveau embrasser du regard des complexes toujours plus grands (une chaîne complète de montagnes, une contrée, tout un pays)".

Janos Kornai [1971], p. 32.

En économie, la question de l'échelle d'observation apparaît pourtant avec le problème du no bridge entre la microéconomie des comportements individuels et la macroéconomie des grands agrégats. Le danger d'un sophisme de composition' ${ }^{9}$, mis notamment en avant par Keynes, est lié à des phénomènes d'émergence ( $c f$. l'exemple de la salle de spectacle), c'est-à-dire précisément à la thématique qui nous intéresse ici. Pourtant, au-delà de ce constat fondamental, la

9 Le sophisme de composition consiste à conclure du tout à partir des parties (croire que ce qui est vrai pour une partie d'un ensemble peut être transposé à l'ensemble) ; celui de la division consiste à l'inverse à conclure des propriétés des parties à partir du tout.

10 Economie et Institutions $-n^{\circ} 8-1^{\text {er }}$ semestre 2006 
question de l'échelle d'observation n'a pas suffisamment retenu l'attention, y compris chez les hétérodoxes d'obédience keynésienne ou autre. Tout au plus pose-t-on la question des fondements micro de la macroéconomie. Le plus souvent, les économistes tendent à se ranger dans les camps antagonistes des partisans de la micro ou de la macro ou à considérer que la question d'échelle se résout avantageusement dans une division du travail entre sous-disciplines.

Or, comme le remarque l'historien Jacques Revel (1996, p. 12), "le problème n'est pas tant ici d'opposer un haut et un bas, les grands et les petits, que de reconnaître qu'une réalité sociale n'est pas la même selon le niveau d'analyse - ou, comme on le dira souvent dans ce livre, l'échelle d'observation - où l'on choisit de se situer. Des phénomènes massifs que nous sommes habitués à penser en termes globaux, comme la croissance de l'Etat, la formation de la société industrielle, peuvent être lus en termes tout différents si l'on tente de les appréhender à travers les stratégies individuelles, les trajectoires biographiques, individuelles ou familiales, des hommes qui leur ont été confrontés. Ils n'en sont pas moins importants pour autant. Mais ils ont été construits autrement".

La construction de l'objet, des faits, diffère selon l'échelle adoptée, du microéconomique au macroéconomique. Il est possible d'en donner un exemple à partir d'un concept économique par excellence : le marché. Qu'est-ce qu'un marché10 ? Au macroscope, c'est la rencontre d'offres et de demandes agrégées impliquant des acteurs institutionnels - au sens de l'INSEE - (Etat, banques, entreprises, ménages) hautement stylisés, agissant au sein de règles générales régulant les échanges. Au mésoscope, le marché est restreint à une branche, il rassemble des organisations échangeant des catégories de biens et services similaires, dans le cadre de règles sectorielles spécifiques. Le niveau de stylisation des acteurs et des produits échangés est moindre. Au microscope, le marché est encore plus restreint, il apparaît comme un ensemble d'organisations très concrètes, reliées par des réseaux, avec des acteurs "en chair et en os " qui échangent des biens et des services spécifiés, impliquant des relations de concurrence, de coopération, de négociation, de pouvoir, le tout au sein de règles et routines organisationnelles pour partie idiosyncrasiques.

Le choix d'une focale dépend également du projet de connaissance du chercheur

La question qui se pose est la pertinence du choix d'une focale d'observation par rapport à un projet de connaissance. Les

10 La question est loin d'être évidente. Rappelons qu'il n'existe pas d'entrée " market " dans le New Palgrave Dictionnary of Economics. 
géographes, au travers de leur expérience cartographique, ont depuis longtemps développé une réflexion sur le sujet. "La généralisation [...] opère par sélection des termes et par perte de la singularité, du détail, de la différence conçue comme secondaire. Les cartographes dans leurs pratiques quotidiennes le savent : généraliser pour pouvoir dessiner une carte, c'est sacrifier des détails du tracé d'un fleuve ou d'une ligne de rivage en fonction de l'échelle de la représentation choisie, c'est diminuer le nombre des variations reconnues comme pertinentes." (Lepetit, 1996, p. 73). "Ainsi, plus qu'un rapport de similitude au réel, l'échelle en désigne une réduction. Elle exprime une intention délibérée de viser un objet et indique le champ de référence dans lequel le réel se pense. L'adoption d'une échelle est d'abord le choix d'un point de vue de connaissance" (ibid., p. 86).

On peut en donner une illustration à partir de la question du découpage sectoriel en économie. La délimitation des contours de la branche s'avère toujours délicate ${ }^{11}$. Pour John Maurice Clark (1961, p. 104), ces difficultés de définition ne peuvent être résolues qu'en interaction avec l'objet de la recherche : "globalement, les arguments en faveur du maintien de concepts comme ceux de secteur ou de groupe semblent plus forts que les arguments en faveur de leur abandon en tant que notion d'orientation. Il convient de reconnaitre que leurs limites sont des questions de degré, à l'origine d'une certaine latitude et doivent être précisées dans les études empiriques en adéquation avec les faits ressortant de chaque cas ". Cette relative plasticité de l'approche sectorielle n'est pas pour autant synonyme de découpages ad hoc: elle résulte de la nécessaire adéquation entre les caractéristiques de la branche (sa construction technico-socioéconomique) et les objectifs théoriques que poursuit le chercheur. Il n'y a pas de bonne limitation en soi, définissable a priori. Ainsi, plus la précision exigée est forte, plus la circonscription du secteur, sa "définition " au sens aussi bien optique que taxinomique du terme, se restreint. Par exemple, l'analyse des modes de gouvernance d'un secteur peut s'appuyer sur les délimitations institutionnelles du secteur. En revanche, l'étude de l'influence des caractéristiques du produit (complexe ou générique, durable ou à consommation répétée, à consommation habituée ou volatile etc.) sur le devenir des entreprises du secteur, nécessite fréquemment d'opérer une différenciation plus fine à l'intérieur de la branche ${ }^{12}$.

11 Il n'est pas possible de rappeler ici les nombreux travaux sur la question du découpage sectoriel. Remarquons simplement avec Hollignsworth et al. (1996, p. 8) qu'"il n'existe pas de définition généralement acceptée des frontières d'un secteur ".

12 On retrouve cette souplesse et ce pragmatisme dans les approches sectorielles de la régulation. Ainsi le secteur agricole est tantôt analysé dans

12 Economie et Institutions $-\mathrm{n}^{\circ} 8-1^{\mathrm{er}}$ semestre 2006 
La question est de savoir dans quel champ de validité l'explication peut se situer en fonction de l'échelle d'observation choisie. S'il existe des échelles plus pertinentes que d'autres pour développer certaines problématiques et tester certaines hypothèses, il faut essayer sur chaque sujet particulier d'établir aussi systématiquement que possible les conséquences provoquées par l'échelle choisie sur le contenu des grilles explicatives.

Figure 2 : Echelle et "visée " de connaissance
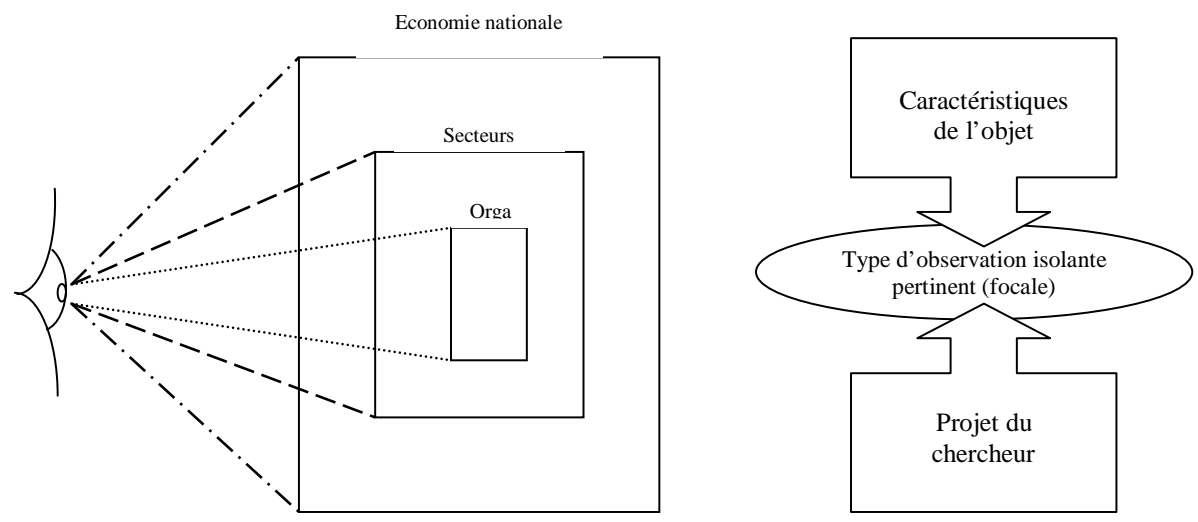

Le principe de variation d'échelles

Non seulement, il importe de développer un questionnement en adéquation avec une échelle d'observation mais il s'avère surtout intéressant de multiplier les échelles d'observation. Il est pertinent, pour reprendre l'image de Kornaï, de faire varier l'altitude d'observation, d'un vol en rase-mottes au plus près des acteurs, à un vol à haute altitude permettant d'embrasser de larges étendues. La variation d'échelles est un principe heuristique, une source de fécondité analytique car elle permet de construire des objets complexes et, selon 1'heureuse expression de Jacques Revel (1996), de prendre en compte "la structure feuilletée du social ". Aucune échelle n'a de primauté ontologique sur une autre : c'est leur mise en regard qui procure le plus fort bénéfice analytique. Ainsi que le souligne Bernard Lepetit (1996, p. 92) "Tout modèle est réduction qui ne retient de l'objet que certaines dimensions. Mais celles-ci ne sont pas en nombre limité, et chaque réduction en appelle d'autres : la recherche est une dynamique sans fin ". Il nous semble que cette

son ensemble, tantôt au travers de découpages plus désagrégés (vins AOC et vins de pays par exemple). Cf. Saillard Y. (1995) et Du Tertre C. (1995). 
option méthodologique peut correspondre à une stratégie praticable d'opérationnalisation des théories de la complexité, à une réponse possible aux défis que pose la notion d'autonomie.

Dans notre travail sur les mutations de l'économie estallemande (Labrousse, 2003) nous avions tenté de mettre en pratique ce principe de variation d'échelle. Ainsi, les changements étaient-ils d'abord scrutés au macroscope : il s'agissait dans un premier temps d'étudier les déterminants macroéconomiques du choc inattendu et inédit qu'a subi l'économie est-allemande après la réunification. L'analyse se fondait sur une vision très stylisée des évolutions et des acteurs. Ensuite, au travers du mésoscope se dessinaient les déterminants des mutations sectorielles dans la diversité des évolutions des modes de gouvernance et des populations organisationnelles : règles d'organisation sectorielles, configurations d'acteurs est- et ouest-allemands mais aussi structures de marché propres à chaque branche. Enfin, le microscope révélait la dimension microéconomique de la transformation. Celle des réseaux interentreprises, celle de frontières et de règles organisationnelles mutantes, celle de l'entreprise " apprenante ". Au fur et à mesure que l'analyse progressait, les variations observées, le degré de diversité des phénomènes augmentait. De nouveaux facteurs explicatifs venaient se rajouter à ceux qui avaient été mis en évidence aux niveaux supérieurs. Ce qui était considéré comme une boîte noire à un niveau, était "décortiqué " au niveau inférieur, le niveau supérieur devenant lui-même une composante de l'environnement.

Evoquer la question des échelles d'observation, c'est déjà soulever la question du regard actif de l'observateur dans la construction de l'objet. Une question qui mérite à présent notre attention.

\subsection{L'autonomie de l'observateur : la nécessité de la réflexivité}

"[...] une description (de l'univers) implique celui qui le décrit (l'observe). Ce dont nous avons besoin maintenant est une description de celui qui décrit ou, en d'autres termes, il nous faut une théorie de l'observateur. [...] cette tâche revient au biologiste. Mais lui aussi est un organisme vivant, ce qui veut dire que, dans sa théorie, il doit non seulement rendre compte de lui-même mais également de la formulation de cette théorie. C'est dans le discours scientifique un nouvel état de choses ; car, en suivant l'optique traditionnelle, qui établit une séparation entre l'observateur et ses observations, toute référence à ce discours était soigneusement évitée ".

Heinz von Foerster (1974, p. 139)

14 Economie et Institutions $-n^{\circ} 8-1^{\text {er }}$ semestre 2006 
L'autonomie des représentations: chaque observateur a un regard propre sur son objet

La question de l'autonomie se pose également pour le chercheur qui ausculte la réalité. Il s'agit de l'autonomie des perceptions, des représentations ${ }^{13}$ par rapport au "réel ", d'une autonomie cognitive. Elle résulte de ce que Foerster appelle les "propriétés de représentation ", liées à la subjectivité et à l'historicité de l'observateur. Aussi existe-t-il autant de vues sur la réalité que d'observateurs. Cependant, ces vues ne sont pas purement contingentes. Le réel existe indépendamment du chercheur qui vise à mieux le comprendre et l'expliquer. Mais ce réalisme est critique et non pas naïf. Le réel ne se donne pas immédiatement à voir. Pour y accéder, un processus de construction de l'objet est nécessaire. Les idées et les représentations sont des constructions sociales en un sens direct; les objets ne le sont qu'en un sens indirect, par l'intermédiaire des idées. Max Weber (1904-1917, p. 205), à l'instar de nombreux institutionnalistes, s'inscrit dans une telle optique constructiviste: "son constructivisme se recommande de Kant, auquel on doit l'idée fondamentale de la théorie moderne de la connaissance' 'selon laquelle les concepts sont et ne sauraient être que des moyens intellectuels en vue d'aider l'esprit à se rendre maître du donné empirique'".

Les idées, les concepts sont des constructions mentales actives, sélectionnées et transformées par l'esprit pour sonder et comprendre la complexité du monde externe. Le chercheur procède à un découpage, par sélection d'un fragment de réalité. Ce découpage correspond à l'" observation isolante " dont parle Schmoller. Eucken (1939), dans la lignée d'Husserl, fait quant à lui référence à une "abstraction pointante et soulignante " (pointierende hervorhebende Abstraktion). Dans cette même veine phénoménologique ${ }^{14}$, Alfred Schütz (cité in Longuet, 2004, p. 4) décrit ainsi la construction de la réalité : "à strictement parler, il n'y a pas de choses, telles que des faits purs et simples. Tous les faits sont d'emblée sélectionnés dans un contexte universel par les activités de notre esprit. Ils sont donc toujours des faits interprétés ou des faits considérés comme détachés de leur contexte par une abstraction artificielle ou alors des faits considérés dans leur organisation particulière. Dans les deux cas, ils portent en eux leur horizon d'interprétation interne et externe. Cela ne signifie pas que, dans la vie quotidienne, ou dans la science, nous soyons incapables de saisir la réalité du monde. Cela signifie simplement que nous n'en saisissons que certains aspects, notamment ceux qui sont pertinents pour nous, soit pour gérer notre

13 Le terme de représentation n'est pas entendu ici au sens de simple reflet du réel, il n'a pas le sens de " mise en présence " immédiate du réel.

14 Schütz est également influencé par Weber, le cercle de Vienne et l'école autrichienne. Plus tard, il s'intéressera au pragmatisme. 
propre vie, soit du point de vue du corpus de règles de procédure de pensée admises telles quelles, appelé méthode scientifique ".

Cette subjectivité de la production de connaissances est enchâssée dans le social, dans des construits collectifs historiquement situés. Philippe Corcuff (1995, pp. 157-158) résume ainsi la pensée de Schütz sur le sujet : "À la base de la connaissance savante du monde social, il y a donc sa connaissance ordinaire: Toute interprétation de ce monde est basée sur une réserve d'expériences préalables, les nôtres propres ou celles que nous ont transmises nos parents ou nos professeurs'; ces expériences, sous forme de 'connaissances disponibles', fonctionnent comme 'schèmes de référence', au sein d'un stock de connaissances disponibles. [...] Le monde que vise la connaissance quotidienne est d'emblée un monde intersubjectif et culturel, parce qu'il n'est pas seulement le mien mais celui d'autres hommes, dont ceux qui m'ont précédé, et qu'il est constitué de significations qui se sont sédimentées à travers l'histoire des sociétés humaines. [...] Ni du côté des acteurs, ni du côté du monde social auquel ils participent, il n'y a homogénéité : $1^{\circ}$ ) le stock de connaissances disponibles n'est pas le même pour chaque acteur : il y a 'distribution sociale de la connaissance', liée à la situation biographiquement déterminée de chacun"; et $2^{\circ}$ ) le monde de la vie quotidienne est structuré en 'diverses couches de réalité, en réalités multiples". Le système de pertinence de l'observateur comprend donc une dimension sociale et historique qu'il s'agisse de la pertinence motivationnelle (intérêt), thématique (questionnement) ou interprétative (volonté de résolution de problèmes). Les thèmes de recherche et leur traitement reflètent pour partie une demande sociale, universitaire, politique.

L'autonomie de l'observateur résulte également de l'autonomie du champ scientifique par rapport à son objet de cognition. "Schütz différencie la connaissance savante du monde social propre au sociologue et la connaissance ordinaire, sur laquelle elle prend appui. Le chercheur en sciences sociales qui observe le monde social est guidé par un système de pertinences différent de celui de l'acteur qui prend part directement à l'action (ce qui est pertinent pour l'un, ne l'est pas nécessairement pour l'autre) ; le savant, qui veut connaître et non agir dans la situation observée, est amené à se détacher d'elle et à puiser, pour ce faire, dans le stock de connaissances disponibles propre à sa discipline scientifique ${ }^{15}$ (son

15 On notera que le système de pertinence de l'observateur dépend étroitement de la discipline dans laquelle il s'inscrit. Ainsi, les rares ethnographes qui s'aventurent dans l'entreprise y retrouvent d'abord les problématiques canoniques de la discipline : don/contre-don, chaud/froid, pur/impur, systèmes de parenté, rites et fêtes et vont délaisser des thématiques telles que les salaires ou les rendements qui font quant à elles

16 Economie et Institutions $-n^{\circ} 8-1^{\text {er }}$ semestre 2006 
corpus de règles de procédures, de méthodes, de techniques, de concepts et de modèles) " (Corcuff, 1996, p. 158). C'est sur ces règles procédurales de la raison scientifique qu'il convient à présent de s'attarder.

Face à la complexité du réel, la rationalité limitée et procédurale du chercheur

"There are more things in heaven and earth, Horatio, than are dreamt of in your philosophy."

William Shakespeare (Hamlet, I-5, pp. 166-167).

" Si nous acceptons qu'à la fois la connaissance et la puissance computationnelle du preneur de décision sont sérieusement limitées, alors nous devons distinguer entre le monde réel et la perception qu'en ont les acteurs et raisonner sur cela. Ce qui veut dire que nous devons construire une théorie (et la tester empiriquement) du processus de décision. Notre théorie doit inclure non seulement le processus du raisonnement mais aussi le processus qui génère la représentation subjective par l'acteur du problème de décision "

Herbert Simon (1997, pp. 368-369)

La notion de représentation, de découpage du réel est incompatible avec les modèles de rationalité qui ne distinguent pas entre le monde réel et la perception qu'on peut en avoir. Or, en dépit de quelques évolutions récentes, cette dimension cognitive est confinée aux marges de l'horizon néo-classique ${ }^{16}$. En revanche, elle

partie intégrante des systèmes de pertinence des sociologues du travail ou des économistes.

16 André Orléan souligne que les représentations, au travers des croyances, ont fait leur apparition dans la théorie standard élargie. Toutefois, cette dimension cognitive n'a pas de retentissement sur le noyau théorique et méthodologique standard. "Si l'on considère la littérature économique des deux dernières décennies, on la trouve présente dans de nombreux travaux, y compris des travaux qui se veulent, par ailleurs, parfaitement 'orthodoxes'. A titre d'exemples, on peut citer les 'équilibres de signalement' (Spence, 1973), les 'bulles rationnelles' (Blanchard et Watson, 1984), les 'équilibres de taches solaires' (Chiappori, 1994), les 'paniques bancaires' (Diamond et Dybvig, 1984) et autres 'prophéties autoréalisatrices' (Farmer, 1999). Dans toutes ces recherches est mis clairement en avant le rôle que jouent les croyances des agents dans la détermination des grandeurs économiques. Pourtant, il ne semble pas que la théorie économique ait pris la pleine mesure de ce résultat. Par exemple, on ne trouve guère d'études empiriques s'attachant à rendre compte de ces représentations, à en préciser la nature et les évolutions. De même, aucune élaboration théorique originale ne s'est interrogée sur la manière dont les conceptions traditionnelles de la valeur se trouvaient alors remises en cause. Tout se passe comme si, faute d'un cadre 
s'insère dans les modèles de rationalité limitée, subjective et procédurale développés par Simon. Appliqués communément aux firmes et aux ménages, ces modèles peuvent être transposés à l'acteur-observateur qu'est le chercheur ( $c f$. tableau 1 en annexe). Si l'on en croit Pat Langley, Simon lui-même avait envisagé cette possibilité dès $1966^{17}$. Les capacités cognitives (et matérielles !) du chercheur sont limitées, il ne peut ni tout lire, ni explorer empiriquement tous les domaines qui semblent pertinents pour son objet. Nulle théorie, aussi complexe soit-elle, ne saurait épuiser la complexité du réel : il est toujours plus de choses sur la terre comme au ciel que n'en rêve notre philosophie. C'est ce que Catherine Colliot-Thélène qualifie, à propos de l'analyse wébérienne, de "postulat d'incomplétude "18. Une théorie n'étant applicable à un objet concret que par l'intermédiaire d'une certaine schématisation, la limitation du pouvoir explicatif de la théorie correspond à la limitation imposée par cette schématisation. Toute la question est de savoir si celle-ci peut être considérée comme acceptable, satisfaisante, par rapport aux problèmes réels que l'on se pose. En effet, si tout modèle est réduction, la méthodologie employée doit être

analytique d'accueil adéquat, cette réalité demeurait invisible, reléguée aux marges de la théorie, à la manière d'une curiosité sans grande importance, voire d'un pur artefact mathématique sans contenu réel ". (Orléan, 2002).

17 "En 1966, Herb Simon publia un chapitre 'Découverte scientifique et la psychologie de la résolution des problèmes' dans lequel il eut l'audace de suggérer que l'on pouvait expliquer et modéliser la découverte scientifique qui comprenait souvent une perspicacité créatrice - en termes de processus cognitifs usuels, suivant des mécanismes similaires à ceux qui sont à la base de la résolution des problèmes humains quotidiens " (Langley, 2004, p. 463). 18 "La présupposition d'un " hiatus irrationalis " irréductible entre concept et réalité (Weber empruntait l'expression au philosophe Emil Lask), est sans aucun doute le présupposé le plus fondamental de l'épistémologie wébérienne $[\ldots]$ Ce hiatus, soit l'impossibilité de rendre compte exhaustivement de tous les éléments qui ont contribué à produire un événement donné, tient à la multiplicité des causalités impliquées en cette production. Pour qu'une explication exhaustive soit possible, il faudrait admettre un état originel dont nous maîtriserions tous les paramètres, et à partir duquel nous pourrions dérouler la chaîne sans lacune de ses effets. [...] Plutôt que d'interpréter la thèse du hiatus irrationalis entre concept et réalité comme une concession aux doctrines irrationalistes, on y verra donc un présupposé gnoséologique que l'on peut nommer le postulat d'incomplétude. La défiance de Weber à l'égard de toutes les variétés de déductivisme est l'envers critique de son pluralisme causal. L'explication historienne ou sociologique doit accepter, si elle veut éviter le dogmatisme, de confesser son caractère inévitablement partiel. L'infinité inépuisable des causalités impliquées dans tout phénomène historique, aussi circonscrit soit-il, oblige à reconnaître que la connaissance de la réalité ne peut jamais être autre chose que celle d'un 'fragment limité' de celle-ci (Essais sur la théorie de la science, p. 153). "(Colliot-Thélène, 2006, p. 27).

18 Economie et Institutions $-n^{\circ} 8-1^{\text {er }}$ semestre 2006 
la moins réductionniste possible au regard du projet de connaissance du chercheur et des caractéristiques de son objet.

Afin de faire face de manière satisfaisante à ces limites, le chercheur met en œuvre des procédures de recherche plus ou moins sédimentées, partiellement transmises par ses pairs, partiellement créées, construites pour s'ajuster aux propriétés de l'objet ${ }^{19}$. Ces procédures de recherche sont enchâssées dans un contexte historique, politique et social. Elles sont largement relatives au cadre institutionnel et organisationnel des organismes et réseaux scientifiques dans lesquels le chercheur s'inscrit. Elles sont relatives à l'état des connaissances du moment. Son projet de recherche passe par des processus d'essais-erreurs ${ }^{20}$. L'observateur se heurte à la résistance du réel. Cette résistance du réel a des effets productifs dans le processus heuristique, elle conduit à mettre en œuvre de nouvelles questions, à faire progresser la démarche par itérations successives. Ces tâtonnements ne sont pas de simples "frottements ", ils sont constitutifs du métier de chercheur, de sa créativité. Ils lui donnent son épaisseur. En cela la recherche est un métier qui s'apprend, nous y reviendrons.

Le raisonnement complexe implique donc de sortir de l'idée d'une séparation objective entre observateur et objet d'analyse. L'observateur s'inscrit dans un processus d'interaction avec son objet. La construction de l'objet participe elle-même d'une situation complexe qui nécessite d'être exposée clairement et d'être soumise à la critique. Robert Delorme (1999) distingue à ce propos la complexité de premier ordre (complexité de l'objet) et la complexité de second ordre (complexité liée au regard de l'observateur). Schütz parle quant à lui de "constructions de second degré ". Ce constat va de pair avec la nécessité de la réflexivité, en rupture avec les approches traditionnelles auxquelles von Foerster faisait allusion. Il implique également le caractère pluriel des rationalités scientifiques, des procédures de recherche. L'acceptation d'une pluralité des points de vue possibles sur la connaissance est une résultante fondamentale de la reconnaissance de la subjectivité du chercheur et du contexte de la recherche. Ainsi que le souligne Geoffrey Hodgson (2001), ce pluralisme est celui de la corporation scientifique dans son ensemble, il ne saurait être un éclectisme incontrôlé au niveau d'un chercheur individuel. Si, bien entendu, le chercheur doit se confronter à ces approches plurielles et les confronter elles-mêmes à son objet, sa démarche est soumise à une exigence de cohérence dont il ne saurait faire l'économie.

19 Sur ce point, comme sur beaucoup d'autres évoqués ici, on se reportera avec profit à l'ouvrage de Bourdieu P., Chamboredon J.-C. \& Passeron J.-C. [1968].

${ }^{20}$ Cf. le bref article de Leontief (1982, p. 23). 


\subsection{L'autonomie des acteurs : la nécessité d'une démarche d'enquête ouverte}

Il nous faut à présent envisager une troisième déclinaison de l'autonomie, liée à la précédente: l'autonomie des acteurs. Les acteurs étudiés, leurs schèmes de perception, leurs catégories d'analyse, leurs motivations, sont partiellement autonomes par rapport à l'observateur. Les acteurs évoluent dans un environnement spécifique et jouent un rôle actif dans la définition même de cet environnement et dans l'interprétation des signaux issus de celui-ci. Selon André Orléan (1998, pp. 24-26), " on peut penser que c'est précisément faute d'avoir intégré pleinement cette dimension à son analyse que l'économie théorique néo-classique ou néo-walrassienne reste impuissante à comprendre de nombreuses situations, comme les bulles financières ou les rapports à la monnaie et à la politique monétaire. Dans de tels contextes, il apparaît avec force que la manière dont les agents économiques interprètent leur environnement est une donnée cruciale des enchaînements économiques. Par exemple, on a souvent observé qu'une même hausse du taux de l'intérêt pouvait conduire à une réaction opposée des marchés boursiers ou des marchés des changes selon que les opérateurs y voient le signe d'une faiblesse ou une promesse de plus grande rentabilité ". Ce "poids des croyances", des représentations renvoie à l'autonomie des acteurs économiques. Or l'individu de l'économie standard, autosuffisant et passif, "donné, isolé et définif ", comme le dit fort bien Veblen (1898), ne saurait être autonome. C'est bien plus un automate à la fois autiste (le social, l'environnement ne le construisent pas $^{21}$ ) et parfaitement hétéronome car entièrement programmé de l'extérieur par les règles du calcul économique standard. Et l'autonomie, nous l'avons vu, suppose simultanément un certain degré d'ouverture et de fermeture. Pour qu'il y ait autonomie de l'individu, celui-ci doit être incomplet, inachevé et capable d'apprentissages. Si ce portrait n'est pas celui de l'homo oeconomicus neoclassicus, il correspond en revanche à celui de l'homo oconomicus institutionnalis, dressé notamment par les institutionnalistes allemands (Schmoller, Weber) et américains (Commons, Veblen). L'individu des institutionnalistes est actif et ouvert tout à la fois, c'est un individu sous influence sociale mais doté d'une réelle personnalité, d'une mémoire, de propensions, d'habitudes, d'intentionnalités et de croyances.

21 On notera que les travaux d'économie expérimentale montrent la présence d'un framing effect dans les processus de choix des individus qui va à l'encontre de cette hypothèse néoclassique. $C f$. Kahneman \& Tversky Ed. (2000).

20 Economie et Institutions $-n^{\circ} 8-1^{\text {er }}$ semestre 2006 
Rationalités locales, connaissances situées et enquête de terrain

De l'autonomie des acteurs, il découle que le chercheur ne peut imaginer a priori les contraintes et les ressources pertinentes dans lesquelles s'enchâssent les décisions des acteurs. Même en supposant à la manière de Laplace que le chercheur connaisse tout de la situation des entreprises ou des ménages qu'il étudie, il ne pourra modéliser ou axiomatiser complètement le comportement des acteurs: c'est la conséquence logique de l'existence d'acteurs autonomes, obéissant à des comportements téléologiques. Les circonstances influant leurs comportements sont d'une très grande variété, elles comprennent des composantes idiosyncrasiques. Elles ne sont pas forcément compliquées ${ }^{22}$ (telle relation personnelle du directeur de l'entreprise étudiée a permis de décrocher un contrat décisif par exemple) mais elles sont souvent déterminantes pour le devenir de l'organisation étudiée. Sur de nombreux aspects, les connaissances des acteurs sur leur domaine d'action sont de très loin supérieures à celle du chercheur. Ces connaissances "situées " des acteurs résultent d'une longue interaction avec leur environnement local. Celles-ci font défaut au chercheur, quelle que soit l'étendue du travail de documentation préalable (on retrouve ici la fragmentation des connaissances et la rationalité limitée du chercheur). D'où la nécessité d'une démarche d'enquête ouverte permettant la remontée d'informations. On peut penser aux enquêtes basées sur des entretiens semi-directifs voire même à l'enquête par observation directe ou participante (Arborio \& Fournier, 1999). Ces méthodes d'enquête, très travaillées en sociologie ou en ethnographie, sont négligées à l'excès en économie ${ }^{23}$. Cet état de fait est probablement lié à la domination du schéma déductifnomologique et aux hypothèses dominantes sur la rationalité des agents qui dispensent de s'interroger et d'interroger les acteurs sur leurs modes de comportement ${ }^{24}$. Quant aux économistes hétérodoxes contemporains, ils accompagnent rarement leur hétérodoxie

22 Selon Simon, la complexité des comportements observés reflète d'abord celle de leur environnement.

23 Pour se convaincre de l'apport de telles approches à l'analyse des organisations économiques, on consultera par exemple Moulinié (1993).

24 N'y aurait-il pas une contradiction foncière entre l'hypothèse de rationalité des acteurs qui caractérise la plupart des modèles et leur reniement en pratique dans une opposition entre la rationalité supérieure des experts (ceux là même qui produisent des modèles) et l'irrationalité des acteurs qui contreviennent aux préceptes des modèles, l'adéquation des comportements au modèle devenant alors le critère de rationalité ? Il y a là une circularité du raisonnement qui pose problème. En outre, que devient le critère de rationalité, si certains acteurs sont plus rationnels que d'autres ? Cela fait éclater l'hypothèse d'homogénéité des acteurs qui est dans bien des cas mais pas toujours - à la base des modèles standards. 
théorique d'une hétérodoxie méthodologique ${ }^{25}$. Il ne s'agit aucunement de renoncer aux outils actuellement en usage en économie (économétrie par exemple) mais de les croiser et les combiner avec des méthodes d'investigation permettant d'intégrer de manière plus ouverte le point de vue des acteurs. Il conviendrait peut-être de réévaluer l'apport des " connaissances ordinaires " 26 , de sonder plus avant les finalités, les rationalités des acteurs. Des économistes hétérodoxes comme Albert Hirschman (1984) avaient déjà attiré l'attention sur les "rationalités cachées " des gens de terrain. Pour Hirschman, ces rationalités qui se distinguent de celles des experts ont leur raison d'être. Elles correspondent à des pratiques sédimentées par l'expérience et potentiellement ajustées à des situations locales. Ces rationalités cachées ne se donnent pas toujours à voir immédiatement. C'est à l'enquêteur de les faire affleurer. Walter Eucken [1939, pp. 10-14] insiste également sur l'importance des vues des acteurs car elles permettent de sortir de la circularité de théories, celles-ci inclinant presque naturellement à l'autoréférentialité. Une démarche d'enquête ouverte permet donc de rompre la circularité, voire l'autisme des théories, d'introduire de nouveaux éléments. Nous verrons qu'une telle démarche s'inscrit avantageusement dans une approche abductive, présente en filigrane dans de nombreux courants institutionnalistes.

Eucken souligne l'apport de ces expériences du quotidien mais aussi leurs limites. En effet, cette rationalité située n'est pas sans contrepartie: en dehors de leur "pré-carré ", beaucoup d'éléments échappent aux acteurs locaux. C'est une implication de la présence d'effets non intentionnels des actions individuelles au sein de systèmes économiques denses ${ }^{27}$. Le chercheur qui dispose de sources systématiques a une vue d'ensemble. Le rapport entre sources systématiques et enquête de terrain est donc fortement complémentaire. Il convient à la fois de prendre au sérieux le sens commun des acteurs tout en étant à même de le dépasser.

25 Ce n'était pas le cas dans la tradition institutionnaliste originaire, tout particulièrement dans l'institutionnalisme américain où l'enquête, dans la lignée pragmatiste de Dewey, avait une importance cruciale.

26 Callon, Lascoume \& Barthes (2001) emploient le terme de "recherche en plein air " à propos des profanes qui s'investissent dans l'investigation de problèmes habituellement réservés aux experts. Pour ces auteurs, l'association des chercheurs en laboratoire et des chercheurs en " plein air " permet l'élaboration de nouvelles manières de voir et l'acquisition de connaissances nouvelles, fondamentales dans un monde incertain.

27 " Sera considéré comme dense tout système d'acteurs où la décision de l'un peut avoir une répercussion non prévue et non intentionnelle sur au moins un autre acteur ", Sapir (1999, p. 193). 
Langage de l'observateur, langage des acteurs : la science, opération de traduction

L'autonomie des acteurs comprend en outre un aspect particulier, l'autonomie de leur langage, notamment par rapport à celui du chercheur. Les ethnologues le savent bien, eux qui ont développé les catégories d'émiques et étiques ${ }^{28}$. Les discours émiques correspondent aux discours des indigènes, objets de l'observation, et les discours étiques aux discours des observateurs-ethnographes ${ }^{29}$. Là encore, l'autonomie est liée au caractère contextuel des pratiques langagières. Le langage des acteurs est inscrit dans des pratiques situées, ce qu'indiquent aussi bien la sociologie du langage que la linguistique pragmatique ${ }^{30}$. En ce sens, l'investigation scientifique correspond à une opération de traduction, à un passage du dit ordinaire au dit savant. Il s'agit d'une reformulation en langage spécialisé (langue cible, souvent écrite) du langage des acteurs (langue source, souvent orale) qui va largement dépendre des objectifs de communication assignés à la discipline. Elle implique le passage d'un registre à un autre, elle est déplacement de sens et de contexte. Comme toute traduction, elle est aussi interprétation, elle implique des transformations et donc des possibilités de trahison, elle participe de la construction scientifique de la réalité. Les acteurs scientifiques et ordinaires se livrent en effet à travers le langage à une activité de typification du monde social (cf. Schütz). Les réponses faites au chercheur ne sont pas dénuées d'ambiguïté. Le chercheur se doit en conséquence de mentionner autant que possible le contexte, de montrer les opérations de transcription, de restituer la pluralité des interprétations possibles. Il s'agit également de mener une double réflexion, une réflexion sur les catégories des acteurs et une réflexion sur les catégories de l'observateur. Nous y reviendrons

28 Ces notions ont été proposées pour la première fois par Pike (1954).

29 L'anthropologue Jackie Bouju (2003) exprime clairement cette idée : "L'ethnographie a pour mission scientifique de rapporter les faits dans les termes de la culture locale. Mais les "faits" caractéristiques du domaine de l'anthropologie sont des "faits de signification". Les hommes, dont l'ethnographe étudie la culture, donnent un sens à ce qu'il font et disent et ils lui en parlent. Or les significations ne se voient pas, aussi convient-il de les extraire par un processus d'abstraction à partir des données recueillies. La description ethnographique de la culture consiste donc en une traduction associée à une interprétation qui se présente comme une synthèse entre la perspective systématique, positive, inductive et comparative dont l'ethnologue est porteur (l'étique) et la perspective singulière, historique et culturelle dont le groupe est porteur (l'émique) ".

30 La linguistique pragmatique s'intéresse aux unités linguistiques dont la signification ne peut être comprise qu'en contexte. Par contexte, il faut entendre le cadre spatio-temporel, l'âge, le sexe des/du locuteur(s), le moment d'énonciation, le statut social des énonciateurs etc. 
ultérieurement à propos de l'intérêt de l'histoire conceptuelle et de l'analyse de la genèse sociale des idées. Dans le même ordre d'idées, l'économiste peut avoir recours avec profit à l'analyse lexicographique ${ }^{31}$. L'économie gagnerait à explorer plus avant ces questions sur lesquelles elle a pris un retard important sur les autres sciences sociales.

Plus généralement, la discipline économique doit mieux prendre en compte l'autonomie de la langue et des catégories de pensée. La langue n'est pas un véhicule neutre permettant d'accéder directement au réel. A l'instar de la monnaie qui n'est pas ce voile qu'il suffirait de lever pour accéder directement aux réalités économiques, la langue n'est pas ce voile neutre derrière lequel des concepts naturels et univoques s'offriraient directement à l'intelligence du réel. Rappelons que les transferts entre théories linguistiques et théories économiques ont été nombreux, et surtout que les théories de la richesse, de la marchandise et de la monnaie ont été, le plus souvent, solidaires de théories sur le signe linguistique (Foucault, 1966, p. 90). Un surcroît de réflexivité en économie sur les catégories d'analyse, que la discipline économique s'exprime au travers de langage naturels (économie 'littéraire') ou formels (mathématiques), est probablement souhaitable.

\section{INSTITUTIONNALISME HISTORIQUE ET ANALYSE SITUEE}

La prise en compte de l'autonomie semble, nous l'avons remarqué à plusieurs reprises, foncièrement incompatible avec l'approche économique standard. En revanche, elle présente une affinité manifeste avec certains courants institutionnalistes. C'est ce lien entre autonomie et théorie institutionnaliste que nous souhaiterions maintenant approfondir.

\subsection{Institutionnalisme historique et réflexivité}

Existerait-il alors une opposition entre deux couples binaires: historicité du social et du chercheur d'un côté et universalisme nomothétique et extériorité du chercheur au monde social de l'autre ? Ce qui frappe éminemment ce sont les affinités électives entre ces approches: au sein de l'économie qui vise à

31 Mbida \& Pairet (2005) remarquent à propos d'une enquête sur les pratiques coopératives des entreprises que le vocable coopération est peu employé par les entrepreneurs pourtant engagés dans de telles pratiques. "Le concept de coopération a davantage une portée académique (analytique) et trouve peu de pertinence pour les praticiens que sont les entrepreneurs ". L'analyse lexicographique a donc conduit à une réinterprétation des réponses paradoxales des acteurs au questionnaire d'enquête et a permis de reconstruire ensuite l'objet.

24 Economie et Institutions $-n^{\circ} 8-1^{\text {er }}$ semestre 2006 
dégager des lois universelles, le processus de construction de l'objet, le regard de l'observateur n'apparaît pas, le chercheur est extérieur à son objet. Les lois dégagées se veulent universelles parce qu'elles sont supposées exister indépendamment des constructions établies par l'observateur pour atteindre le réel. De manière symétrique, dans l'institutionnalisme historique, la modestie de l'objectif - l'analyse de régularités situées dans le temps et dans l'espace - s'accompagne d'une historisation de l'observateur scientifique. C'est particulièrement frappant chez Veblen, Commons, Weber ou encore Myrdal. Cette conjonction de facto de l'historicité de l'objet étudié et de l'historicité du chercheur indique-t-elle quelque chose de plus fondamental qui tiendrait à la logique même des postures ? Les deux aspects vont-ils nécessairement de pair?

La contextualisation de la recherche, objet ou ruine de la science économique?

Il y a en tous cas un lien étroit. De même que les régularités historiques qu'il dégage, l'observateur est historicisé32. C'est aussi pour cette raison que les lois dégagées ne sauraient être universelles. A l'inverse, pour préserver le caractère universel des lois économiques, l'économie standard est contrainte de postuler la neutralité du chercheur. Il y a symétrie entre les hypothèses gouvernant les agents économiques et les présupposés - plus ou moins explicites - quant à la position de l'économiste. Si les acteurs sont omniscients, si les processus de découverte peuvent être négligés, alors qu'en est-il du métier de chercheur? N'est-il pas in fine superflu?

Mais la posture historicisante ne ruine-t-elle pas finalement le principe même d'une science économique? Telle fut en tous cas l'opinion de nombre de contemporains français de l'école historique allemande. Elle reste celle d'une majorité d'économistes contemporains. L'objection mérite donc d'être prise au sérieux. On peut, à titre d'exemple, mentionner la réaction de Gide \& Rist (1922, p. 436) à l'égard de l'un des représentants de l'ancienne école historique: "Hildebrand conteste l'existence même des lois économiques naturelles, telles que les concevaient les classiques. Il

32 Knies (cité par Gide \& Rist, 1922, pp. 464-465) formule cette idée de manière tout à fait explicite: "De même que les conditions de la vie économique, dit-il, de même la théorie économique, quels que soient sa forme et son aspect, les arguments et les résultats qu'elle nous offre sont un produit du développement historique, elle emprunte le fond de son argumentation à la vie historique et doit donner à ses résultats le caractère d'une solution historique : même les 'lois générales' de l'économie ne sont autre chose qu'une explication historique et une manifestation progressive de la vérité ". 
reproche à Roscher d'en avoir admis l'existence. Par cette affirmation hasardée, Hildebrand ne paraît pas s'être aperçu qu'il ruinait le principe même de toute science économique [... "33. L'idée aristotélicienne qu'" il n'y a de science que du général " et que la subjectivité de l'observateur disqualifie immanquablement son travail continue de traverser la science économique. En quoi les productions d'un chercheur "situé " peuvent-elles avoir le statut de connaissances " vraies "?

Il convient de noter au préalable que la contextualisation du chercheur n'implique pas nécessairement une posture post-moderne et relativiste, elle vise ici au contraire à développer des techniques d'objectivation. La lecture post-moderne de l'économie en fait un discours au même titre que le roman. Elle finit par dissoudre l'économie dans la fiction. Même si l'économie comme toute science sociale opère une "mise en intrigue " qui la fait entrer dans l'ordre $\mathrm{du}$ récit et du discours, l'homologie ou, plus exactement, la parenté des figures discursives n'implique pas une identité épistémologique entre le discours économique et la fiction. Il est tout à fait possible de définir un espace original qui évite le double écueil du relativisme et du positivisme. De nombreux développements récents au sein des science studies s'efforcent précisément de délimiter cet espace étroit et incommode, "au bord de la falaise " (Chartier, 1998), entre prise en compte du contexte historique des sciences (dures) et production de connaissances vraies ${ }^{34}$.

\section{Dépasser la subjectivité du chercheur : les règles du métier}

Il faut alors donner toute sa mesure à la spécificité épistémologique du discours scientifique, à ses " règles du métier "35. Ce sont précisément les opérations spécifiques et les procédures de validation et de contrôle, mentionnées plus haut qui rendent possibles l'existence d'un tel espace ${ }^{36}$, qui permettent de répondre à la gageure d'un constructivisme sans relativisme. Marc Bloch parle du métier d'historien, Weber (1919) traite de la science comme métier

33 Leur position n'est pas isolée : ainsi André Liesse affirme-t-il que l'école historique, " nouveau jouet venu d'Allemagne ", "part de ce principe, elle a du moins celui-là, que la science économique n'existe pas ". Compte rendu de l'ouvrage d'Adolphe Houdard : Premiers principes de l'économie politique, Journal des économistes, janvier 1890.

${ }^{34} C f$. le $\mathrm{n}^{\circ}$ spécial de la revue Mouvements, ${ }^{\circ} 17$, sept-oct. 2001 sur le thème "A plusieurs voix sur Entre science et réalité - La construction sociale de quoi?".

35 On trouvera des développements extrêmement intéressants sur la notion de métier appliquée à la recherche en science sociale chez Bourdieu, Chamboredon et Passeron (1968).

36 Cf. Certeau M. de (1974).

26 Economie et Institutions $-\mathrm{n}^{\circ} 8-1^{\mathrm{er}}$ semestre 2006 
(Wissenschaft als Beruf). Métier car les pratiques de recherche sont faites de routines, d'habitudes incorporées et transmises, de procédures de recherche éprouvées dont la pertinence peut et doit toujours être questionnée. Il y a une sédimentation d'héritages, de gestes techniques, de choix méthodologiques, de modèles d'intelligibilité à partir de supports spécifiques au métier. Il est remarquable que Veblen [1899-1900] parle des " habitudes mentales du chercheur " comme il étudie les habitudes dans les entreprises. Il y a une spécificité de chaque métier mais des processus communs liés à l'apprentissage, la pratique et la transmission de savoirs dans un cadre organisé, régulé. C'est pourquoi il semble pertinent d'appliquer les instruments généraux forgés dans l'analyse d'autres milieux à l'univers social de la recherche ${ }^{37}$, comme le fait Veblen. Il en est de même de Commons qui en appelle à " la nécessité de l'autoréflexivité des [chercheurs en sciences sociales] en tant qu'ils font partie eux-mêmes du monde qu'ils étudient " (Théret B., 2001). On notera à ce propos la fécondité d'une approche dans laquelle la réflexion épistémologique prend place dans un va-et-vient avec l'analyse des objets. L'idée d'un métier d'économiste a pour corollaire que les pratiques de recherche ne sont pas réductibles à des énoncés théoriques a priori, qu'un mouvement de balancier entre réflexion méthodologique et pratiques théoriques est indispensable. Il n'y a pas d'étanchéité entre le monde du chercheur et le monde social mais des champs distincts du social dont le champ scientifique est une composante. Il convient donc d'articuler l'enchâssement des pratiques scientifiques dans le social avec la spécificité des règles du métier de chercheur. Il existe donc bien une logique qui unit les hypothèses des théories économiques sur le réel et les théories de la connaissance que portent ces théories. On mentionnera au passage que les approches orthodoxes sont singulièrement muettes sur cette question 38 .

37 Gérard Noiriel (1998) dans un entretien avec Roger Chartier note à ce propos : "que l'une des faiblesses persistantes des sciences sociales tient au fait que les chercheurs qui s'en réclament ne parviennent pas vraiment à éclairer leur propre univers social en mobilisant les instruments qu'ils ont forgés pour étudier les autres milieux".

38 Il semblerait en effet que parmi les économistes standards, on ne pense guère à appliquer ses propres catégories d'analyse à la production de connaissances au sein de la discipline économique. Friedman, dans son célèbre article de 1953 sur la méthodologie de l'économie positive, exclut la science économique du champ d'application de l'économie dans une rhétorique de l'inspiration : "La construction d'hypothèses est un acte créatif d'inspiration, d'intuition et d'invention. [...]. Ce processus doit être discuté au travers de catégories psychologiques et non logiques, étudié au travers des biographies et des autobiographies et non de traités sur la méthode scientifique " (Friedman, 1953, pp. 42-43). Gary Becker (1975) est plus cohérent à cet égard. 
La construction de l'objet ne fait pas seulement référence au produit final de la recherche, à son architecture finie, elle renvoie également au processus de construction lui-même ${ }^{39}$. C'est ce processus qu'il convient de mettre en lumière et d'exposer à la critique. Il s'agit de donner à voir les conditions de l'observation, de la production des "données", de replacer les catégories et les concepts employés dans leur contexte afin de mieux situer le travail et d'en permettre une lecture critique. Il s'agit par là de mettre en œuvre des procédures d'objectivation qui font partie intégrante des règles du métier précédemment évoquées. Nous en proposons ici quelques unes, allant de l'histoire de la pensée à l'auto-analyse, en passant par l'étude de la genèse sociale des catégories et des concepts.

La fonction réflexive de l'histoire de la pensée

A l'heure actuelle, la réflexivité de la discipline économique sur ses propres pratiques, sur ses fondements épistémologiques, semble circonscrite au cercle étroit des spécialistes es questions méthodologiques. Cette lacune est d'autant plus manifeste qu'elle est loin de valoir pour toutes les sciences sociales. A titre d'exemple, l'exercice de la réflexivité est particulièrement présent parmi les historiens. Ces derniers disposent d'ouvrages tels que les Ecrits sur l'histoire de Fernand Braudel, le Comment on écrit l'histoire de Paul Veyne, l'Apologie pour l'histoire de Marc Bloch, La Nouvelle Histoire de Jacques Le Goff etc., dont on serait bien en peine de trouver les pendants en économie. Tous ces écrits sont le fait de praticiens de la discipline et non d'épistémologues professionnels. L'historiographie constitue en outre une source majeure de réflexivité, exercée collectivement au niveau de la discipline historique.

En économie, l'équivalent fonctionnel de l'historiographie est l'histoire de la pensée économique. Comme ailleurs, il existe une pluralité d'approches en la matière (Lapidus, 1996). Celles approfondissant l'historicité de la discipline mériteraient peut-être plus d'attention qu'elles n'en ont actuellement. L'histoire des idées économiques in retrospect de Mark Blaug (1978) qui lui permet de dédier son ouvrage de référence "à son fils Ricardo " tient encore le devant de la scène. Or, une telle vue aboutit in fine à une interprétation profondément ahistorique de l'histoire de la discipline. Seule est alors pertinente la contemporanéité des ancêtres dont la

39 Ce double sens a été mis en évidence par Ian Hacking (2001).

28 Economie et Institutions $-n^{\circ} 8-1^{\text {er }}$ semestre 2006 
pensée est mesurée à l'aune des canons de la discipline telle qu'elle s'offre à nous aujourd'hui. Mais ce n'est pas toujours le cas.

L'institutionnalisme historique allie réflexion en histoire de la pensée et théorisation des problèmes concrets. Ainsi, Schmoller développe ses réflexions méthodologiques en les inscrivant dans l'histoire de la pensée économique. Commons en fait une source majeure dans la construction de son système de pensée à côté, notamment, de l'histoire et de l'expérience des faits. Hirschmann a conjugué étroitement travaux en histoire de la pensée et en économie du développement. Plus récemment, Hodgson souligne l'importance de l'histoire de la pensée comme réservoir d'idées pour les économistes du présent. Une telle conception implique une vision non linéaire de l'histoire des idées avec des trajectoires diversifiées et accidentées, des bifurcations et des arrêts, des oublis, des silences et des bégaiements. Elle s'oppose à l'idée d'une progression linéaire vers un perfectionnement toujours plus grand d'une science économique tendant mécaniquement vers une efficience allocative optimale des recherches. Si tel était le cas, il serait effectivement superflu et même contre-productif d'aller fureter chez les anciens dans une perspective autre que celle de l'antiquaire, leur pensée ne formant que le maladroit balbutiement de celle des modernes.

On perçoit encore une fois combien conception du monde social et conception du monde scientifique sont liées. Il serait sans doute judicieux de développer cette fonction réflexive à l'histoire de la pensée. Pourquoi l'économiste ne ferait-il pas une petite histoire de la pensée portant sur son objet empirique et sur sa boîte à outils théorique ? Il pourrait y puiser des sources d'inspiration et resituer les mouvements récents qui agitent le microcosme des sciences économiques. Une telle incursion, modeste et ciblée, en histoire de la pensée ne viendrait pas supplanter l'histoire de la pensée des spécialistes de cette sous-discipline mais la compléter par des interrogations issues de "pratiques théoriques ". Une telle démarche permettrait d'injecter une dimension réflexive au cœur même des études empiriques.

\section{L'historicisation de l'observateur : auto-analyse, égo-histoire}

D'autres formes de réflexivité, à un niveau plus individuel cette fois, sont envisageables. A titre d'exemple, l'" ego-histoire "40, un genre émergent en histoire depuis la fin des années 1980, né dans un contexte d'ébranlement des repères traditionnels de l'objectivité historique, pourrait constituer une source d'inspiration pour les

40 Cf. l'ouvrage collectif Nora (1987) avec des contributions, outre celle de l'éditeur, de Maurice Agulhon, Pierre Chaunu, Georges Duby, Raoul Girardet, Jacques Le Goff, Michelle Perrot et René Rémond. 
économistes. L'historien y replace dans une perspective historique ses propres travaux. Il retrace son itinéraire, analyse les inflexions, les détours, les tâtonnements, les abandons qui ont jalonné ses recherches ainsi que les arènes, les réseaux, les influences qui ont façonné sa production scientifique ${ }^{41}$. Selon la formule de Marc Bloch, il livre "le spectacle de la recherche, avec ses succès et ses traverses $\star^{42}$. Loin de proposer un récit égotiste, une réflexivité toute narcissique, l'ego-histoire se distingue du récit biographique, des mémoires : elle n'est pas une confession, c'est l'historien et non sa personne qui en est l'objet, un historien enchâssé dans des institutions et des organisations scientifiques, politiques et sociales. Cela permet de replacer un travail par rapport à l'évolution des paradigmes et modes scientifiques, à des effets de génération, à la demande sociale et politique qui s'exerce autour de l'objet de la recherche. Les préférences politiques et sociales de l'observateur, son système de valeurs, méritent d'être explicités. Si ces préférences ne déterminent pas directement le traitement du sujet, elles jouent un rôle moteur dans le choix des thématiques, dans la motivation du chercheur, dans son expérience vécue et son contact avec la réalité. Cela a été souligné par de nombreux travaux institutionnalistes, en particulier ceux de Gunnar Myrdal (1930, 1958). C'est également le cas de John Commons qui a étroitement mêlé, on le sait, recherche scientifique et participation à la vie politico-économique de son pays $^{43}$. Si le chercheur dit "d'où il parle ", s'il expose honnêtement la progression de son travail telle qu'elle a pris place, il rend possible la lecture critique de ses travaux. A quand l'ego-économie?

Certains courants ethnographiques proposent de pratiquer l'auto-analyse qui n'est pas sans similitudes avec l'ego-histoire ${ }^{44}$. Florence Weber (1996, p. 8) la définit comme l'" analyse réflexive des pré-notions de l'ethnographe en tant qu'il est l'indigène d'une culture

${ }^{41}$ La pratique de l'ego-histoire s'institutionnalise aujourd'hui en France. Elle devient la règle dans les soutenances d'habilitation à diriger des recherches en histoire. Signe de son succès, elle porte simultanément en germe le risque d'en faire une simple convention narrative, étape obligée d'une carrière.

42 "Tout livre d'histoire digne de ce nom devrait comporter un chapitre, ou si l'on préfère, insérée aux points tournants, une suite de paragraphes qui s'intitulerait à peu près : 'comment puis-je savoir ce que je vais dire.' Je suis persuadé qu'à prendre connaissance de ces confessions, même les lecteurs qui ne sont pas du métier éprouveraient un vrai plaisir intellectuel. Le spectacle de la recherche, avec ses succès et ses traverses, est rarement ennuyeux. C'est le tout fait qui répand la glace et l'ennui " (Apologie pour l'histoire, p. 68, cité in Weber, 1996).

43 A cet égard, l'autobiographie de Commons, parue la même année que ses Institutional Economics, s'avère particulièrement éclairante. Merci à l'un des rapporteurs d'avoir attiré notre attention sur cet ouvrage.

44 On notera que l'auto-analyse en ethnographie ne fait pas référence à la notion psychanalytique éponyme.

30 Economie et Institutions $-n^{\circ} 8-1^{\text {er }}$ semestre 2006 
liée à sa position sociale et à son appartenance au monde savant ". Pour elle (1996, p. 9), "loin d'être une oiseuse introspection, cette auto-analyse est objectivation ". L'auto-analyse vise non seulement à une distanciation par rapport à des pré-notions mais, plus fondamentalement, à mettre au jour le processus de construction de l'objet. Deux pratiques sont particulièrement intéressantes à cet égard : " le journal d'enquête, carnet de bord de l'observation directe où sont consignés au jour le jour les évènements tels que mon regard les perçoit, les trie, les choisit $[\ldots]$; le journal de recherche où s'élaborent parallèlement les interprétations successives qui devront converger dans la construction finale". Pour Florence Weber, ils constituent "une source, une archive de l'ethnographe, au même titre que des archives produites par d'autres ou que des retranscriptions d'entretiens". Cette source permet en outre de mettre à jour les perturbations du " milieu " qu'apporte le chercheur, de mieux comprendre l'interaction enquêteur/enquêté. En effet, "l'enquête est une interaction sociale d'un type particulier et non seulement une technique de recueil des données " (Weber, 1996, p. 19).

Dans une perspective cognitive, de telles démarches procèdent non seulement des techniques d'objectivation du savoir mais fournissent aussi des ressources, une mémoire vive des procédures de recherche, dans laquelle la communauté savante pourrait puiser. A l'instar du management de la connaissance dans les départements de R\&D des entreprises, où est conservée une mémoire des inventions non abouties ou non retenues, en vue d'éventuelles utilisations dans un autre contexte, les trajectoires de recherche devraient donner plus souvent lieu à publication et être intégrées aux parutions classiques. Il y a en outre une véritable créativité des chemins de traverse, des stratégies du détour dans l'investigation scientifique. Cela serait particulièrement utile pour les apprentis chercheurs. Ces incursions au cœur de la fabrique de la recherche fourniraient des exemples moins intimidants que le résultat final et figé des recherches. Ce "management de la connaissance " scientifique permettrait un meilleur apprentissage du métier de chercheur et une circulation des connaissances moins biaisées.

Il convient donc de sortir de la vision héroïque du chercheur, génie solitaire et inspiré décrit par Milton Friedman ou de l'économiste-expert purement objectif, pour pénétrer dans la cuisine quotidienne de la recherche. La posture institutionnaliste ne va-t-elle pas de pair avec l'humilité de l'économiste, chère à André Orléan (2001)? 
Nous avons déjà envisagé à plusieurs reprises la prise en compte des catégories de perception de l'observateur. L'histoire fournit ici aussi des outils intéressants - est-ce bien étonnant? pour comprendre la genèse sociale des idées. Pensons en particulier à l'histoire des concepts, la Begriffsgeschichte ${ }^{45}$. Loin d'une simple étude linguistique de concepts détachés de leur contexte social, la Begriffsgeschichte s'inscrit dans l'histoire sociale. Il s'agit d'" une histoire langagière des concepts, attentive aux échanges incessants entre langue et société et aux écarts entre des usages actuels et des usages passés d'un même concept, étant entendu que tout maniement actuel d'un objet d'étude passé implique une histoire des concepts qui ont permis de le nommer" (Hartog, 1997). La sémantique historique est considérée à la fois comme "indicateur et facteur de changement social "46.

Or, selon Ian Hacking (2001), ce qui caractérise les sciences sociales, c'est précisément l'existence d'une interaction entre les concepts qu'elles développent et les individus ou les comportements classés ; en qualifiant une personne ou un comportement, on peut l'affecter, et même le transformer. Dans les sciences de la nature en revanche, une telle interaction ne saurait exister : développer tel ou tel concept de quarks est sans effet sur ce qu'ils font et sur ce qu'ils sont. Cette interaction entre catégories des sciences sociales et objet d'étude devient elle-même un élément de l'analyse. Ce caractère performatif est particulièrement flagrant pour un matériau fondamental en économie: les statistiques. On dispose aujourd'hui de nombreux travaux sur la construction sociale et historique des statistiques en France et ailleurs ${ }^{47}$. Les statistiques sont à la fois un outil de connaissance et de décision. Leurs modalités de construction influencent les résultats. Il s'agit de s'interroger sur la genèse sociale et politique des catégories statistiques afin d'en permettre un usage plus averti, mais aussi affiner les catégories existantes et dégager des informations nouvelles. Alain Desrosières (1992) propose de "discuter l'indiscutable ", à savoir le chiffre et sa production. Il s'agit en outre de sortir les études statistiques du statut de simple "méthodologie subordonnée" (Desrosières, 2003) à la théorie économique. Sur ce thème, Nicholas Balabkins rapporte que

45 L'historien allemand Reinhard Koselleck a été le principal initiateur de l'histoire des concepts (Begriffsgeschichte, l'expression est de Hegel). Sur la notion de Begriffsgeschichte, cf. Koselleck (1986) et le numéro spécial "Materiali per un lessico politico europeo : ,storia dei concetti' ", de Filosofia politica, $\mathrm{n}^{\circ} 3$, décembre 1987.

46 Avant-propos à l'édition française du Futur passé de Koselleck [1979], Editions de l'EHESS, Paris, (1990), p. 15.

47 Cf. notamment Desrosières A. (1993).

32 Economie et Institutions $-n^{\circ} 8-1^{\text {er }}$ semestre 2006 
Schmoller demandait à ses étudiants de produire dans un premier temps eux-mêmes le matériau statistique nécessaire à leur travail afin de prendre conscience de la nature d'une telle production et de ne pas séparer recueil de données et processus de théorisation ${ }^{48}$. Pourquoi ne pas réintroduire un tel enseignement aujourd'hui en économie?

Historiciser l'objet : une causalité cumulative, séquentielle et circulaire

"La référence à l'histoire ne se réfère [sic] pas à une succession d'évènements qui prirent place hier mais évoque plutôt les processus par lesquels le temps transforme une multiplicité de futurs possibles en un passé unique [...] Le hasard, la nécessité et la volonté humaine forment une trilogie de création et de destruction, de hasard et de permanence, d'adaptation et de pétrification".

Jacques Lesourne (1994, p. 25)

L'économie qu'étudie le chercheur est située dans le temps et dans l'espace. L'autonomie des différentes composantes des systèmes économiques implique l'historicité et l'hétérogénéité du social. Les évolutions de l'environnement sont retraduites, retravaillés par des processus internes à ses composantes tissant ainsi des trajectoires singulières, idiosyncrasiques. La science ne doit alors pas se contenter de penser le général. Le singulier s'avère également un objet scientifique légitime ${ }^{49}$. Nous touchons là un point central de la pensée historiste en économie, en particulier celle de Max Weber : "spécificité, particularité, singularité, unicité, autant de termes équivalents pour identifier le projet de connaissance propre des sciences historiques, qui appelle des instruments gnoséologiques différents de ceux dont disposent les sciences du général, concepts génériques ou lois. La critique du 'monisme naturaliste', c'est-à-dire

48 "Schmoller fit, avec ses collègues, du séminaire une nouvelle forme d'enseignement [...] Aussitôt que les thèmes des travaux étaient répartis, Schmoller exigeait que leur traitement s'adosse méticuleusement à un matériau statistique. Il exigeait des étudiants qu'ils collectent eux-mêmes ce matériau, en allant dans les archives et en se familiarisant avec les documents anciens ou, en produisant eux-mêmes par exemple le chiffre des accidents du travail dans les entreprises. Ce n'est qu'ensuite que les participants au séminaire avaient l'autorisation de confronter le matériau collecté avec celui déjà disponible dans les revues et ouvrages spécialisés " Balabkins (1993, p. 22),

49 Weber (1904-1917, p. 294, note) souligne que ce caractère singulier, historique ne constitue pas le differencia specifica de la discipline historique: il concerne "toute imputation 'historique' de n'importe quel événement singulier, y compris ceux de la nature inerte. La catégorie de 1'historique' est prise ici comme un concept logique et non comme un concept technique propre à une spécialité ". 
de la conception selon laquelle le but de toute science devrait être de formuler des lois générales, représente ainsi un moment fondamental de l'épistémologie wébérienne "(Colliot-Thélène, 2006, p. 19). Penser le singulier n'implique pas d'abdiquer de tout concept de causalité, loin de là. Weber parle à ce propos d'imputation causale singulière.

L'approche est généalogique, genèse et fonction devant être distinguées. Le rejet du fonctionnalisme ${ }^{50}$ est une caractéristique centrale de l'institutionnalisme historique qui le différencie de la nouvelle économie institutionnelle. Il s'agit d'éviter une vision téléologique de l'évolution des sociétés, une idée centrale chez Schmoller, Weber, Commons, Veblen ou encore Boyer aujourd'hui. La causalité est cumulative et séquentielle. Le temps est tissé de phénomènes d'irréversibilité51 de degré variable. Il n'est ni homogène, ni continu, ni causalement inerte (Sapir, 2000). La notion de dépendance du sentier suivi (David, 1985; Arthur 1989) saisit l'existence de phénomènes d'irréversibilité, de non-linéarité dans l'évolution économique. Les changements sont cumulatifs au point que peuvent apparaître des phénomènes de verrouillage (lock-in). Ils sont aussi marqués par la contingence et l'aléatoire. De petits évènements initiaux, souvent fortuits, peuvent se trouver amplifiés par la dynamique des processus cumulatifs ${ }^{52}$. Ces questions sont depuis longtemps travaillées par les courants institutionnalistes de List à Boyer en passant par Veblen ${ }^{53}$. Le caractère séquentiel de la causalité s'applique également aux anticipations, aux croyances des acteurs économiques. Cela implique de distinguer, à la suite de Myrdal, entre l'ex-post et l'ex-ante.

La causalité est non seulement séquentielle, elle est également circulaire et récursive, avec un retour de l'effet sur la cause. Cette causalité complexe ${ }^{54}$ renvoie à l'" upward and

50 Il y a fonctionnalisme quand l'existence d'un élément du système est totalement expliquée par sa fonction (présente). Gould et Lewontin (1984) ont qualifié une telle approche de Panglossianisme.

51 Cf. Boyer R., Chavance B. \& Godard O. Ed. (1991).

52 Cette dimension a souvent été négligée dans les études empiriques se réclamant de la notion. Le repérage de ces facteurs nécessite en effet fréquemment une analyse désagrégée, localisée dans le cadre de ce que l'on pourrait qualifier de "dépendance du sentier située ". Cf. Labrousse (2003).

53 Cela tient pour partie à la question du Sonderweg allemand du XIXe, c'està-dire de sa trajectoire d'industrialisation spécifique, qui a été l'une des préoccupations fondatrices de ces théories. C'est le cas de Friedrich List, l'un des précurseurs de l'institutionnalisme historique. C'est le cas des écoles historiques et notamment de Werner Sombart dans Der moderne Kapitalismus. C'est également le cas de Veblen [1915], en particulier dans son ouvrage sur la révolution industrielle en Allemagne où se trouve une première théorie de la dépendance du sentier.

54 On peut opposer la causalité complexe à la causalité linéaire où une cause entraîne simplement un effet.

34 Economie et Institutions $-n^{\circ} 8-1^{\text {er }}$ semestre 2006 
downward causation" entre les différentes composantes des systèmes économiques étudiée par les institutionnalistes américains. Elle va de pair avec une approche en terme d'holindividualisme ou encore d'individualisme méthodologique complexe (Reynaud 1997), situationel ou institutionnaliste qui " ne prétend pas que les seules 'causes' en histoire sont le comportement des individus, mais plutôt que ce sont les seules entités au comportement finalisé " (Boyer, 1992, p. 124). Les interactions interindividuelles produisent des phénomènes collectifs dont les propriétés ne se rencontrent dans aucun des individus ou des éléments en cause (Dupuy, 1994, p. 175$)^{55}$. On retrouve ici la nécessité d'une analyse multiscalaire. Cette conception de l'individu conjugue individualité forte et enchâssement historique et social de cette individualité. Schmoller critiquait ainsi l'homme moyen de Quételet qui traite une population où certaines caractéristiques moyennes ont été observées comme si elle était homogène et comme si on pouvait attribuer ces caractéristiques à n'importe quelle partie de la population ${ }^{56}$. L'individu néo-classique obéit quant à lui à des lois telles que sa subjectivité disparaît ${ }^{57}$. C'est un "homme sans qualités ", c'est-à-dire

55 André Orléan en tire les conséquences quant à l'analyse des représentations sociales : "S'il en est ainsi, il nous faut alors reconnaître que le niveau des représentations sociales possède une logique propre, partiellement déconnectée des opinions privées, ce qui constitue une critique du modèle individualiste, de type bottom-up, qui pense l'opinion collective comme somme des opinions individuelles. Cela a de grandes conséquences théoriques et empiriques. D'une part, l'analyse des croyances sociales telles que nous les définissons conduit à une conception forte du collectif, non réductible aux éléments qui le constituent. Dans notre approche, le collectif trouve à s'expliquer d'abord par le collectif, et non par l'individuel. D'autre part, dire que les croyances sociales sont autonomes, c'est leur reconnaître le statut de tiers médiateur, en surplomb par rapport aux interactions individuelles ". Orléan A. (2002), op. cit.

56 C'est un autre représentant de l'école historique, Wilhelm Lexis, qui poussera plus loin l'analyse en remettant en cause l'hypothèse de l'urne unique de composition constante qui constitue la justification théorique de l'homme moyen. Il montre que les séries de Quételet exhibent le plus souvent des variabilités supérieures à celles qui résulteraient du tirage dans une telle urne. Galton et Pearson développeront ensuite des constructions statistiques capables " de rendre compte des distributions et des dispersions et non plus seulement des moyennes ". Cf. Desrosières (1999, pp. 876).

57 C'est en en ce sens, qu'il faut comprendre la phrase de Pareto [1908, p. 260] : "L’individu peut disparaître pourvu qu'il nous laisse cette photographie de ses goûts " Une telle affirmation ne trouve son bien-fondé que dans un monde stationnaire et atemporel dans lequel la photographie a une validité perpétuelle. 
sans singularités 58 . A ce sujet, Schmoller (1911, p. 306) parle de l'u utopique présupposé d'hommes identiques". Cette variabilité du vivant a été théorisée au travers de la notion de population thinking dans le cadre de la biologie de l'évolution ${ }^{59}$. La variation n'y apparaît plus comme un simple accident, produit d'une copie imparfaite d'un même modèle, comme chez Quételet. Elle constitue au contraire une caractéristique fondamentale du monde vivant. Cependant, les institutionnalistes s'accordent sur ce point, cette diversité des individus n'exclue en rien l'existence de régularités statistiques ${ }^{60}$. Pour Schmoller (1869), elles ne sont pas le produit d'une nature humaine atemporelle ${ }^{61}$, elles sont en premier lieu le fruit historique et évolutif des cadres institutionnels. Ces derniers modèlent les comportements sans pour autant les déterminer directement. On retrouve aujourd'hui cette idée phare de l'institutionnalisme historique dans la théorie de la régulation (Billaudot, 1995, p. 209) : "la dynamique macroéconomique n'est soumise à aucune loi générale : celle qui est observable dans tel pays, à tel moment, est relative aux institutions en place. Si ces dernières revêtent des formes qui ont durci au cours d'une certaine période historique, ce système de formes induit un ensemble de régulations partielles, stabilisées dans leurs modalités (délai d'ajustement, degré d'indexation)". Les individus et les institutions interagissent et évoluent dans le temps. Ils sont des construits historiques et non des données exogènes et figées.

58 Desrosières (1999) mentionne à la suite de Jacques Bouveresse que Musil a été fortement influencé par Quételet et les débats allemands sur l'homme moyen.

59 Selon Ernst Mayr (2002, p. 26), "le concept de biopopulation apparaît aujourd'hui comme la différence la plus fondamentale entre le monde vivant et non vivant. Le monde non vivant (inerte) est composé de classes platoniques faites d'essences ou de types dont les membres sont respectivement identiques. La variation apparaît alors comme aléatoire et non pertinente. En revanche, dans une biopopulation chaque individu est unique (einzigartig) et la valeur moyenne d'une population n'est qu'une abstraction. Sur six milliards d'hommes, il n'en existe pas deux qui soient complètement semblables. Les populations d'une classe (Art) ne se différencient pas de manière typologique mais simplement au travers de leur valeur statistique moyenne".

60 On notera que l'école historique a contribué au remarquable essor des catégories et des enquêtes statistiques en Allemagne. Cette assise statistique constitue une caractéristique de l'école historique alors même que, comme le remarque Alain Desrosières (2003), " de leur côté, les théoriciens de l'économie de marché comme Jean-Baptiste Say, Augustin Cournot ou Léon Walras ont été réticents à l'utilisation des statistiques économiques pour étayer leurs développements hypothético-déductifs".

61 C'est l'un des points qui l'oppose à Menger dans le Methodenstreit (Labrousse, 2007).

36 Economie et Institutions $-n^{\circ} 8-1^{\text {er }}$ semestre 2006 
A cette vision historicisée de l'économie répond une vision de l'espace économique où jouent les causalités cumulatives, les polarités. Les travaux de François Perroux (1961) mais aussi de Gunnar Myrdal (1957) l'illustrent bien. Les économies d'agglomérations sous-tendent la concentration géographique de la production. L'espace $\mathrm{y}$ est fondamentalement hétérogène. Le territoire fabrique des actifs spécifiques, actifs qui ne sont pas transposables d'un lieu à un autre. Les hommes et les choses ne sont pas aussi mobiles que dans les modèles néo-classiques. Ils sont souvent "attachés" à des lieux. Les acteurs sont situés au sens fort $\mathrm{du}$ terme. La proximité spatiale importe. Les processus d'apprentissage collectifs sont localisés (Rallet \& Torre, 1995). On retrouve donc la problématique de la spécificité. Historicité et spatialité vont de pair. C'est pourquoi, aux visions standards du temps linéaire, homogène correspondent des visions de l'espace tout aussi homogène, un espace plat et sans rugosité, un espace géométrique qui n'a, en fin de compte, pas de dimension propre. Dans les approches institutionnalistes, les dimensions temporelles et spatiales ne constituent pas un cadre homogène et exogène mais des facteurs constitutifs des dynamiques économiques.

L'analyse comparative apparaît alors comme un outil crucial de théorisation. Une comparaison qui incorpore les cas atypiques. Dans un cadre théorique intégrant d'emblée la question de la spécificité, l'exceptionnel n'est pas rédhibitoire, l'atypique n'est pas à écarter hors du champ d'analyse. Au contraire, il a un intérêt heuristique, notamment quand il est mis en regard avec des régularités. Comme le souligne remarquablement Bruno Théret (1997), dans une approche morphologique ou sociétale, les cas extrêmes ou "déviants" font partie intégrante du système d'explication : ils ne sont pas considérés comme relevant d'un degré résiduel d'erreur mais jouent au contraire un rôle crucial dans la construction du modèle interprétatif. Les différents objets sociaux (organisations, systèmes productifs, système de relations industrielles etc.) ne sont pas comparables terme à terme d'un pays à l'autre car ils ne tirent leur sens que du contexte systémique dans lequel ils sont immergés ${ }^{62}$. En revanche, on peut comparer des

62 La dimension comparative est également présente dans l'analyse standard mais elle repose sur un postulat universaliste qui lui confère un tout autre statut. Les analyses de la croissance économique en fournissent un exemple: "l'analyse conventionnelle suppose que les paramètres de la croissance sont identiques d'un pays à l'autre. Loin d'être un exercice statistique positiviste, ces régressions inter-pays sont liées à un présupposé néo-classique sous-jacent. Chaque pays singulier, selon cette vue, va apporter des preuves (evidence) qui pourront être utilisées pour élucider la relation économique universelle sous-jacente " (McCartney, 2006). Une telle approche se heurte selon l'auteur à la fragilité et à l'hétérogénéité des 
ensembles relationnels définis par un jeu d'interdépendances. Chaque ensemble économique fait système et il est particulièrement délicat d'isoler rigoureusement les variables explicatives (Cross, 1982). La diversité n'apparaît plus comme une aberration mais comme un élément constitutif du social. Il s'agit, au travers de l'approche comparative (variation des niveaux d'analyse, des localités etc.) d'opérer des montées (et descentes) en généralité. L'objectif est d'allier la prise en compte de la singularité avec des processus de montée en généralité, en dépassant ainsi l'opposition réductrice entre approches idiographiques et approches nomothétiques. Il est possible de définir des degrés de généralités relatifs et, dans une proportion exactement inverse, de spécificité. Cela permet de définir les domaines de pertinence et de validité des théories.

Il est donc fondamental de préciser les coordonnées spatiotemporelles des phénomènes qu'éclairent les théories, de les situer. Faute de quoi, le risque est grand d'universaliser un modèle spécifique. Ainsi, à ses débuts, la théorie de la régulation, au travers du concept de fordisme, avait généralisé l'expérience d'un pays, les Etats-Unis, et d'un secteur, le secteur automobile. Elle a ensuite " rectifié le tir " et fait progresser d'autant son cadre théorique. Ces coordonnées sont tridimensionnelles : elles renvoient à des niveaux d'observation différents (organisationnel, sectoriel, macro), à leur inscription dans l'espace (local, régional, national) et le temps (court, long).

\subsection{Une construction abductive de l'objet}

"Le passage des théories de la rationalité substantive aux théories de la rationalité procédurale nécessite un changement fondamental de style scientifique, le passage d'une insistance sur le raisonnement déductif avec un strict système d'axiomes à une insistance sur l'exploration empirique et minutieuse des algorithmes complexes de la pensée".

Herbert Simon (1997, pp. 368-369)

De la critique wébérienne du monisme naturaliste au changement de style scientifique prôné par Simon, l'institutionnalisme en appelle à une logique alternative d'investigation scientifique. On peut tenter d'esquisser un "institutionnalisme méthodologique". Celui-ci repose sur une heuristique de recherche relevant d'un processus abductif.

coefficients de régression observés. Il en conclut que les processus de croissance diffèrent dans le temps et l'espace et plaide pour des études de cas, suivies dans un second temps d'un usage raisonné des régressions statistiques.

38 Economie et Institutions $-n^{\circ} 8-1^{\text {er }}$ semestre 2006 


\section{Abduction et institutionnalisme méthodologique}

De nombreux travaux insistent aujourd'hui sur le lien étroit qui unit institutionnalisme américain, pragmatisme et abduction63. Pour Commons et Veblen, "Les 'théories' ne sont ni des structures logiques déductives, ni des copies inductives du réel mais des outils mentaux de recherche (Commons) ou dispositifs provisoires de travail (Veblen) dans un processus continu de recherche en réponse à l'expérience" (Bazzoli \& Dutraive, 2006, p. 129). Cette remise en cause de la dichotomie induction-déduction, déjà présente chez Schmoller (Labrousse, 2007), confère une place toute particulière à l'observation dans le processus de théorisation ${ }^{64}$. C'est une des caractéristiques de la logique d'investigation développée par le philosophe pragmatiste John Dewey ${ }^{65}$, un auteur qui a fortement influencé les économistes institutionnalistes et évolutionnaires, de même que Peirce. Les hypothèses y sont fondées empiriquement et non pas a priori, le rapport au réel n'intervient pas en bout de course, contrairement au schéma classique déduction-prédictionvérification. Si le recueil des faits est pour partie formaté par les préconceptions de l'observateur, ce formatage n'est pas complet: des faits saillants, surprenants apparaissent. Ils apparaissent d'autant mieux que le chercheur obéit à la règle de "brasser large " dans un premier temps et emploie des techniques d'enquête ouverte, permettant le surgissement de l'inattendu. Il y a alors " résistance "

63 L'abduction est l'inférence, à partir d'un fait surprenant, d'une proposition qui constitue l'hypothèse la plus plausible permettant d'expliquer ce fait. Elle prend la forme suivante : le fait surprenant $\mathrm{C}$ est observé; si A était vrai, $\mathrm{C}$ s'expliquerait comme un fait normal; partant, il est raisonnable de soupçonner (présumer) que A est vrai (Peirce [1905], p. 189). Il est particulièrement difficile de définir en quelques mots l'abduction comme forme d'inférence à côté de l'induction et de la déduction. Pour Tony Lawson (1997), la déduction peut être illustrée par le passage de l'assertion (claim) générale que 'tous les corbeaux sont noirs' à l'inférence particulière que le prochain corbeau que je verrai sera noir; l'induction est quant à elle le passage de l'observation particulière de nombreux corbeaux noirs à l'assertion générale que 'tous les corbeaux sont noirs'; le raisonnement abductif est indiqué par le passage de l'observation de nombreux corbeaux noirs à une théorie d'un mécanisme caractéristique des corbeaux qui les dispose à être noirs.

64 Des auteurs contemporains comme Nelson et Winter (1982, pp. 46-47) le soulignent également.

65 Abraham Hirsch (1998, p. 102) relève sur ce point l'originalité de la logique de Dewey par rapport à celle de Mill "sa logique encourage une observation étendue employant les cadres théoriques disponibles ou qui semblent en affinité avec les observations. Une telle observation étendue est considérée comme souhaitable car Dewey encourageait le raisonnement des faits observés vers les concepts théoriques et ensuite vers de nouvelles observations". 
du réel aux préconceptions de l'observateur. Cette surprise est centrale dans le processus abductif, elle en est le point de départ. Au lieu de considérer ces faits surprenants comme de simples anomalies par rapport au schéma théorique, il convient de les prendre au sérieux et d'en formuler une théorie explicative. Potentiellement, ces " écarts " pointent des lacunes, des blocages au niveau théorique (on retrouve ici l'intérêt des cas atypiques). Cela nécessite de réexaminer les hypothèses initiales, d'affiner les concepts. Ce renouvellement du cadre théorique entraîne de nouvelles observations.

On peut alors définir une série d'itérations entre observation et cadre théorique: préconceptions guidant une définition préliminaire de l'objet - recueil étendu de faits stylisés - apparition d'éléments saillants et surprenants - formulation d'hypothèses explicatives, élaboration d'un cadre théorique pertinent - déduction d'éléments devant intervenir dans le réel selon le modèle - retour à l'observation etc. Le schéma déductif-nomologique est remplacé par la triade récursive A-D-I (abduction-déduction-induction). En effet, l'abduction est incomplète, elle permet simplement de proposer une explication plausible. On passe alors au stade suivant du processus d'enquête : la déduction. La déduction consiste selon Peirce à déduire les conséquences de l'hypothèse explicative née de l'abduction. Les conséquences déduites de l'hypothèse fournissent matière à l'induction d'une règle possible qui reste à expérimenter ${ }^{66}$.

La théorie apparaît comme une matrice exploratoire, nécessitant toujours de se plonger dans des données locales, spécifiques. Elle est processuelle, évolutive, jamais achevée. Elle est le produit de tâtonnements, de bifurcations, de reconsidérations des solutions envisagées. On est en présence de trial and error economics pour reprendre la jolie formule de Leontief (1982), loin des blackboard economics moquées par Coase (1994). La recherche correspond au processus de search défini par Simon, à une forme de rationalité procédurale, délibérative ${ }^{67}$. Ces notions d'abduction et de rationalité procédurale ont le mérite de lier logiques d'investigation et de découverte scientifique, contre Friedman et surtout Popper. Pour ce dernier, il n'existe pas de méthode logique pour avoir de nouvelles

66 Cf. Kruijff G.-J. M. (2005). Dans une telle perspective, l'induction sert à vérifier et non à initier une explication ce qui l'éloigne de Hume et, par la même occasion, du fameux " problème de l'induction ".

67 La pensée de Simon, par son accent sur la démarche et les procédures de recherche, peut être rapprochée du pragmatisme de Peirce (Le Moigne 1994, p. 151). Symétriquement, Peirce part d'hypothèses (incomplétude de la recherche, principe d'économie en temps, énergie et argent dans la sélection des hypothèses à tester, etc.) proches de la rationalité limitée Simonienne. Les liens entre les deux approches mériteraient cependant d'être explorés plus avant.

40 Economie et Institutions $-n^{\circ} 8-1^{\text {er }}$ semestre 2006 
idées, chaque découverte trouvant sa source dans un moment irrationnel, intuitif. L'abduction permet, selon Peirce, d'introduire de nouvelles idées ${ }^{68}$. Elle vise à expliquer de nouveaux faits (Koriche \& Sallantin, 1999, p. 2). Elle est donc en adéquation avec les théories institutionnalistes qui s'intéressent à l'émergence endogène de la nouveauté au travers de processus historiques. Symétriquement, l'hypothèse de nomenclature des biens et des états du monde ${ }^{69}$ de la théorie standard est cohérente avec la méthode déductivenomologique. En effet, pour que cette dernière soit valide, il faut admettre que l'économiste (et l'ensemble des acteurs économiques) dispose de cette description préalable des états du monde ${ }^{70}$. Pour qu'elle ait un caractère prédictif, il convient d'être en présence d'un monde fini et stationnaire, immédiatement et intégralement accessible à l'observateur. La déduction est tautologique et le calcul porte sur un ensemble clos de données, sur des objets déjà constitués. En revanche, la logique délibérative et abductive ne se réduit pas à la mise en place de formules computationnelles, " elle élabore et invente, en partie au moins, les données qui entrent dans la formulation de la décision "(Kechidi, 1998, p. 437). Plongée dans un monde évolutif, soumis à la nouveauté, la contingence et l'incertitude radicale, elle ne saurait déboucher sur des prédictions mais sur des prévisions circonspectes et circonstanciées, des scenarii pluriels. Son objet est d'abord de reconstruire a posteriori $^{71}$ les

68 "L'abduction est le processus de formation d'une hypothèse explicative. C'est la seule opération logique qui introduise une nouvelle idée; car l'induction en fait rien d'autre que la détermination d'une appréciation et la déduction développe simplement les conséquences nécessaires d'une pure hypothèse ". (Peirce, [1905] (1935), p. 171).

69 "Carlo Benetti et Jean Cartelier (1980) ont défini ainsi l'hypothèse de nomenclature des biens : 'L'hypothèse de nomenclature revient à supposer possible une description d'un ensemble de choses, qualifiées de biens ou de marchandises, antérieurement à toute proposition relative à la société'. L'hypothèse de nomenclature des états du monde renvoie au postulat walrassien selon lequel l'incertitude du futur peut-être représentée (et réduite) sous la forme d'une liste de $n$ évènements exogènes ou états du monde, censée décrire de manière exhaustive tous les évènements susceptibles de se produire" (Orléan, 2004).

70 C'est peut-être en ce sens qu'on peut comprendre l'exclamation de Weber (1905, p. 373) : "pourquoi en effet s'intéresser à 1'histoire', si celle-ci se contente de montrer que, au fond, 'tout a déjà existé'? ".

71 Weber distingue de manière intéressante la Vorausberechenbarkeit, sorte de prédictibilité ou calculabilité prospective, de la Berechenbarkeit, forme de "prévisibilité rétrospective" selon C. Colliot-Thélène (2001, p. 154). Pour Weber, les sciences historiques ne sont capables que de prévisibilité rétrospective. C'est lié pour lui au caractère contingent et à l'incertitude non probabilisable propre aux phénomènes historiques. Cette imprédictibilité n'exclue pas l'existence d'un ordre intelligible sous-jacent et l'imputation causale. Weber anticipe à cet égard sur les théories du chaos. Pour celles-ci, 
processus causaux "par lesquels le temps transforme une multiplicité de futurs possibles en un passé unique " selon la formule de Lesourne.

\section{Investigation scientifique et principe de satisficing}

Quand ce processus d'investigation prend-il fin ? Il faut bien à un moment ou un autre une décision d'arrêter la récursivité à l'infini de l'explicitation. Une réponse peut être donnée en appliquant le principe de satisficing de Simon, lié à la notion de rationalité limitée et procédurale, au chercheur lui-même. Selon Pat Langley (2004, pp. 467-468), "Herb Simon utilisait la notion de satisficing au-delà d'une construction théorique utile, il l'évoquait régulièrement comme une heuristique pour sa propre recherche". A quoi peut-on alors reconnaître une explication satisfaisante ? On peut considérer comme indice de satisficing une situation de recherche où la surprise s'estompe, où de nouveaux éléments viennent s'intégrer au cadre théorique existant sans le bouleverser. Peirce [1905, p. 197] répond d'ailleurs de la sorte à la question "qu'est-ce qu'une bonne abduction ? ": outre d'expliquer les faits, "sa fin, au travers de la soumission au test de l'expérience, est de conduire à l'élimination de toute surprise, à l'émergence d'une habitude d'expectation positive qui ne soit pas déçue ".

Ce satisfecit n'est pas le seul fait du chercheur, il s'inscrit dans un processus de délibération critique au niveau de la communauté scientifique. Le travail du chercheur individuel devient un élément, une étape provisoire dans la production collective de connaissances. "C'est pourquoi, la connaissance scientifique est le produit d'un processus d'auto-correction des croyances par la critique de la communauté face à l'expérience, dans lequel la vérité n'est pas de l'ordre de la correspondance à une réalité qui préexisterait à la pensée : la réalité étant évolutive, la vérité n'est qu'une 'limite idéale', comme le dit Peirce, d'une connaissance en perpétuel développement car l'expérience s'étend et se transforme " (Bazzoli \& Dutraive, 2005, p. 16).

Le travail du chercheur est soumis à la critique et à la révision de ses pairs mais aussi, le cas échéant, à celle des acteurs étudiés. Le chercheur peut, en effet, opérer dans certains cas une restitution aux acteurs ${ }^{72}$. Avec son lot de discussions, d'argumentations et de contre-argumentations de la part des intéressés, la restitution soumet la production scientifique à une

déterminisme et imprédictibilité ne sont pas les deux termes d'une opposition simple mais peuvent être associés sans contradiction aucune (théorie du chaos déterministe).

72 Selon les types de terrains, la restitution n'est pas toujours possible ou présente une utilité réduite. 
forme de contrôle par les acteurs eux-mêmes. Plus que l'étape ultime de la recherche, elle constitue un élément du dispositif d'investigation. "En somme, la restitution des résultats, c'est-à-dire leur communication aux intéressés et leur discussion, fournit une autre façon de comprendre le système. C'est en quelque sorte une manière de structurer des entretiens collectifs qui permettent à l'analyste de pénétrer plus avant dans la compréhension de structures profondes du contexte d'action analysé. Par là, elle devient un instrument pour approfondir un certain nombre d'hypothèses sur les structures et les règles du jeu du système qu'on étudie, et pour faire ressortir d'autres 'faits' structurants qui n'avaient pas été vus ou dont la signification réelle avait échappé de prime abord " (Friedberg, 1992, p. 323).

\section{Conclusion}

" En somme, tout déterminisme est partiel, particulier, régional. Il est saisi à un point de vue spécial, dans un ordre de grandeur désigné, dans des limites explicitement ou tacitement fixées ".

Gaston Bachelard (1951, p. 300)

Comme le dit Alain Desrosières (1993, p. 1), "les techniques et les outils mobilisés pour identifier, décrire et expliquer sont historiquement et socialement situés. Lorsque l'on regarde le monde, il arrive un moment où il est utile d'enlever les lunettes et d'en examiner les foyers". Pour Commons (1934, cité in Bazzoli \& Dutraive, 2005, p. 3), les sciences sociales doivent rendre compte de "deux schèmes conceptuels, [celui du chercheur] par lequel se construit sa science, et ceux des êtres humains, son objet, qui se construisent pour leur propre but ". Une logique forte unit à notre avis les hypothèses sur le réel et le procès de connaissance présents au sein des courants relevant de l'institutionnalisme historique. La rationalité située, limitée, procédurale des acteurs économiques et des chercheurs, la causalité cumulative, séquentielle et circulaire et la démarche abductive de construction de l'objet forment le socle cohérent de l'institutionnalisme méthodologique. Ce petit texte constitue un plaidoyer pour une méthodologie non réductionniste mettant en œuvre les principes de variation d'échelle, de réflexivité et d'abduction afin de rendre compte de l'autonomie des niveaux d'observation, du chercheur et des acteurs. Le tableau 1 figurant en annexe en propose une synthèse.

Les ressources sont nombreuses pour développer cet institutionnalisme méthodologique, encore peu formalisé et synthétisé aujourd'hui. On peut les trouver aussi bien dans l'histoire de la discipline économique que dans les sciences sociales voisines. En effet, les premiers institutionnalistes avaient bien plus approfondi 
ces questions méthodologiques et épistémologiques qu'on ne le suppose généralement. Cette tradition mérite d'être réévaluée et revisitée. De plus, l'économie hétérodoxe a l'avantage, largement sous-estimé, d'être compatible avec de très nombreux courants en sciences sociales (Nelson \& Winter, 1982). Les disciplines voisines restent cependant trop souvent considérées implicitement ou explicitement comme des sciences auxiliaires fournissant de simples matériaux pouvant être passés à l'auguste moulinette théorique de l'économiste. Or les trajectoires de ces disciplines leur permettent de bénéficier d'une expérience solide, sédimentée sur plusieurs décennies quant aux questions qui nous occupent ici. Expérience qui fait souvent défaut à la discipline économique marquée par la domination du schéma déductif-nomologique. Il est donc temps de sortir de l'autisme de la discipline économique en développant un véritable dialogue méthodologique interdisciplinaire. L'enjeu fondamental d'un tel échange avec les auteurs passés et avec les disciplines voisines est d'expliciter et de développer une méthodologie alternative cohérente et opératoire qui pourrait être enseignée et transmise. Cette insistance sur les processus d'apprentissage et de transmission des savoirs est au demeurant en harmonie avec la conception d'un métier d'économiste avec ses règles et ses savoirfaire spécifiques.

En parlant d'institutionnalisme méthodologique nous souhaitons mettre l'accent sur la méthode car elle nous paraît centrale. Aujourd'hui, la théorie standard est sans doute moins soudée par un cadre théorique unitaire que par ses préceptes méthodologiques. Si elle reste dominante, elle s'est en effet largement balkanisée au profit d'un patchwork de théories standards élargies à géométrie variable: on a ainsi assisté depuis les années 1970 à l'abandon progressif de la théorie générale au profit d'une multitude de théories particulières (théorie des jeux, équilibres partiels), au relâchement simultané de certaines hypothèses et à l'introduction progressive de nouvelles (théories de l'information imparfaite, des tâches solaires, de la rationalité limitée etc.). Pourtant, peu d'auteurs prennent la mesure de l'ébranlement occasionné qui met en péril la cohérence de l'édifice néo-classique. Si la théorie standard avait une forte cohérence interne, il n'en est plus de même de ses avatars récents. Que reste-t-il finalement de commun aux tenants de l'approche néo-classique actuelle sinon certains axiomes méthodologiques ${ }^{73}$ ? Au sein des approches dites hétérodoxes, il est fréquemment fait usage de la méthodologie standard, ne serait-ce que parce qu'elle a valeur de norme de scientificité quasi-exclusive dans le champ. Ce faisant, les hétérodoxies subissent une forme de

73 Tony Lawson voit ainsi dans la méthode "mathématico-déductive " le noyau dur de la théorie néo-classique.

44 Economie et Institutions $-n^{\circ} 8-1^{\text {er }}$ semestre 2006 
violence symbolique. En effet, les systèmes d'instrumentation ne sont pas indépendants des théories, ils les incorporent largement ainsi que le montre la philosophie des sciences (notion de theoryladeness). Il est donc périlleux d'opérer une séparation étanche entre méthodologie et théorie. Les théories hétérodoxes, faute de déployer une méthodologie alternative à celle du mainstream, ne pourront se développer pleinement et sans contradictions foncières. 
Tableau 1 : Autonomie, complexité et institutionnalisme méthodologique

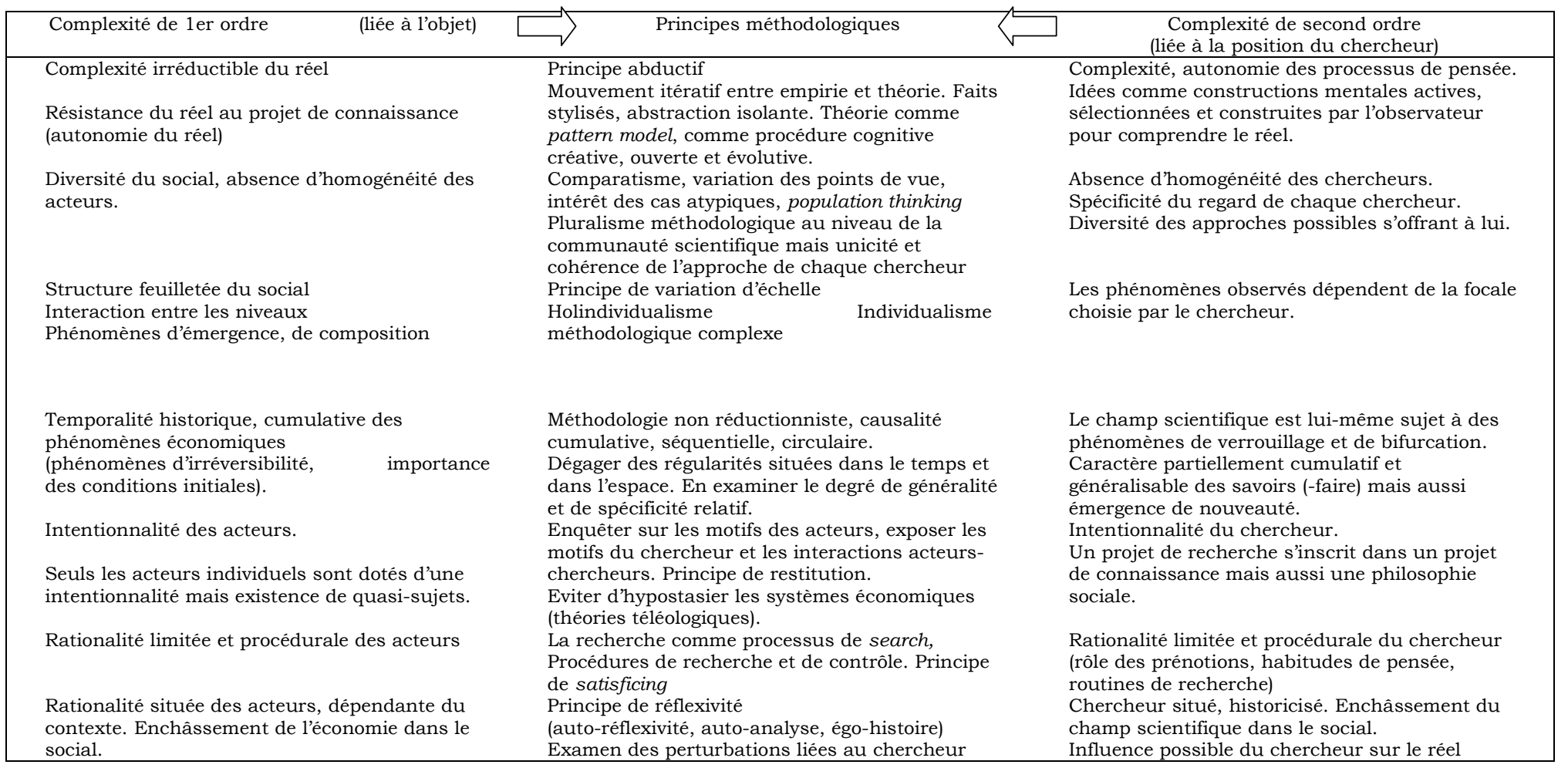

Economie et Institutions $-\mathrm{n}^{\circ} 8-1^{\mathrm{er}}$ semestre 2006 


\section{BIBLIOGRAPHIE}

Arborio A.-M. \& Fournier P. (1999), L'enquête et ses méthodes: l'observation directe, Paris, Nathan.

Arthur W. B. (1989): "Competing Technologies, Increasing Returns, and Lock-In by Historical Events " Economic Journal, 99, pp. 116-31. Atlan H. [1972]: L'organisation biologique et la théorie de l'information, Paris, Hermann, nouvelle édition augmentée (1992), p. 58.

Bachelard G. (1951), L'activité rationaliste de la physique contemporaine, Paris, Presses Universitaires de France, p. 300.

Balabkins N. W. (1993), "Schmoller und der Stammbaum der nationalökonomischen Wissenschaft : 'Mit der Theorie allein kommt man nicht aus' ", in: Backhaus J. G. (1993) : Gustav Schmoller und die Probleme von heute, Duncker und Humblot, Berlin, pp. 19-26.

Bazzoli L. \& Dutraive V. (2005), "Fondements pragmatistes de l'institutionnalisme en économie - Théorie de la connaissance et théorie de l'action chez Veblen et Commons ", Document de travail du LEFI, Lyon.

Bazzoli L. \& Dutraive V. (2006), "Fondements pragmatistes de l'institutionnalisme en économie - Théorie de la connaissance et théorie de l'action chez Veblen et Commons ", Revue de Philosophie Economique, $\mathrm{n}^{\circ} 13$, vol 1, pp. 123-153.

Becker G. (1975), "The University Professor as a Utility Maximizer and Producer of Learning, Research and Income ", Journal of Economic Literature, 17, pp. 503-502.

Billaudot B. (1995), "Formes institutionnelles et macro-économie ", in Boyer R. \& Saillard Y. Ed. (1995), pp. 209-214.

Blaug M. (1978), Economic Theory in Retrospect, Cambridge, Cambridge University Press.

Bouju J. (2003), "La culture dogon : de l'ethnologie coloniale à l'anthropologie réciproque contemporaine ", Clio en Afrique - Bulletin d'anthropologie et d'histoire africaine, $\mathrm{n}^{\circ} 10$, été 2003.

Bourdieu P., Chamboredon J.-C. \& Passeron J.-C. [1968], Le métier de sociologue, Berlin - New York, Mouton de Gruyter.

Boyer A. (1992), L'explication en histoire, Lille, Presses Universitaires de Lille.

Boyer R. \& Saillard Y. Ed. (1995) : Théorie de la régulation. L'état des savoirs, Paris, La Découverte.

Boyer R., Chavance B. \& Godard O. Ed. (1991), Les figures de l'irréversibilité en économie, Paris, Editions de l'EHESS.

Braudel F. [1976], La dynamique du capitalisme, Paris, Flammarion, (1988).

Callon M., Lascoume P. \& Barthe Y. (2001), Agir dans un monde incertain. Essai sur la démocratie technique, Paris, Seuil. 
Certeau M. de (1974), "L'opération historiographique ", in Le Goff J. \& Nora P. (1974), L'écriture de l'histoire, t. 1, Nouveaux Problèmes, pp. 63-120.

Chartier R. (1998), Au bord de la falaise. L'histoire entre certitudes et inquiétude, Paris, Albin Michel.

Clark J.M. (1961), Competition as a Dynamic Process, Washington, The Brooking Institutions.

Coase R. (1994), Essays on Economics and Economists, Chicago, University of Chicago Press.

Colliot-Thélène C. (2001), Etudes wébériennes. Rationalités, histoires, droits, Paris, Presses Universitaires de France.

Colliot-Thélène C. (2006), La sociologie de Max Weber, Paris, La Découverte.

Commons J. R. [1934], Institutional Economics. Its Place in Political Economy, The University of Wisconsin Press, 1959, (1 ed. 1934, Macmillan).

Commons J.R. (1934), Myself, New York, The Macmillan Company.

Corcuff P. (1995), Les nouvelles sociologies : constructions de la réalité sociale, Paris, Nathan, pp. 57-58.

Cross R. (1982), "The Duhem-Quine Thesis, Lakatos and the Appraisal of Theories in Macroeconomics ", Economic Journal, 92, pp. 320-340.

David P. (1985) : "Clio and the Economics of Qwerty", American Economic Review, Proceedings 75.

Delorme R. (1999), "Complexity and Evolutionary Theorizing in Economics ", Paper prepared for the International Workshop on the Evolution and Development of Evolutionary Economics, Brisbane, 1215 juillet.

Desrosières A. (1992), "Discuter l'indiscutable. Raison statistique et espace public ", Raisons Pratiques, 3, 1992, pp. 131-154.

Desrosières A. (1993), "La statistique entre le langage de la science et celui de l'action ", Correspondances.

Desrosières A. (1993), La politique des grands nombres, histoire de la raison statistique, Paris, La Découverte, 1993.

Desrosières A. (1999), "Statistique ", in Lecourt D., pp. 874-880.

Desrosières A. (2003), "Historiciser l'action publique: l'Etat, le marché et les statistiques ", in Laborier P. \& D. Trom, Ed. (2003), Historicités de l'action publique, Paris, PUF, pp. 207-221, passim.

Du Tertre C. (1995), "La dimension sectorielle de la régulation ", in Boyer R. \& Saillard Y. (1995), op. cit., pp. 313-331.

Dupuy J.-P. (1994) : Aux origines des sciences cognitives, Paris, La Découverte.

Eucken R. [1939] : Die Grundlagen der Nationalökonomie, 6e éd., Berlin-Heidelberg, Springer-Verlag.

Favereau O. (1989), "Marchés internes, marchés externes ", Revue Economique, n², pp. 273-328.

48 Economie et Institutions $-n^{\circ} 8-1^{\text {er }}$ semestre 2006 
Foerster H. v. (1974), "Notes pour une épistémologie des objets vivants ", in Morin E. \& M. Piatelli-Palmirini, Ed. (1974), L'Unité de l'homme, t. 2, Le cerveau humain, Paris, Seuil, 1974, pp. 139-155.

Foucault M. (1966), Les mots et les choses. Une archéologie des sciences humaines, Paris, Gallimard.

Friedberg E. (1992), Le pouvoir et la règle, Paris, Seuil.

Friedman M. (1953), "The Methodology of Positive Economics ", Essays in Positive Economics, Chicago, Chicago University Press.

Gide C. \& Rist C.(1922), Histoire des doctrines économiques, Paris, Sirey, 4e édition.

Gould S. J. \& Lewontin R. (1984), "The Spandrels of San Marco and the Panglossian Paradigm: A critique of the Adaptationist Programme ", in Sober E., Ed. (1984), Conceptual Issues in Evolutionary Biology, Cambridge (Mass).

Hacking (2001), Entre science et réalité, la construction sociale de quoi ?, trad. par B. Jurdant, Paris, La découverte.

Hartog F. (1997), "Reinhart Koselleck, lumineux théoricien de l'Histoire ", Le Monde des livres, 28 novembre 1997.

Hirsch A. (1998), "John Dewey ", in Davis et al. (1998), The Handbook of Economic Methodology, Cheltenham, Edward Elgar, pp. 100-103.

Hirschman A. O. (1984), "La confession d'un dissident. Retour sur 'La stratégie du développement économique', in Meier G. M. \& Seers D. Ed. (1988), Les pionniers du développement, Paris, Economica, pp. 93-119.

Hodgson G. (2001), How Economics forgot History, London, Routlege. Hodgson G. (1998), "Emergence ", in Davis J.B., Wade Hands D. \& Mäki U. Ed. (1998), The Handbook of Economic Methodology, Cheltenham, Edward Elgar, pp. 156-160.

Hollingsworth J.R., Schmitter P.C. \& Streeck W. (1994), "Capitalism, Sectors, Institutions and Performance ", in Hollingsworth J.R., Schmitter P.C. \& Streeck W. Ed. (1994), Governing Capitalist Economies, New York-Oxford, Oxford University Press, pp. 3-16, p. 8. Kahneman D. \& A. Tversky Ed. (2000), Choices, Values, and Frames, Cambridge, Cambridge University Press.

Kechidi M. (1998), "Rationalités et contextes de décisions : un retour sur Herbert Simon", Revue Internationale de Systémique, vol. 12, n45, pp. 419-440.

Koriche F. \& Sallantin J. (1999), "Abduction ", in Lecourt (1999), op. cit., pp. 1-4.

Kornai J. [1971]: Anti-equilibrium. A gazdasági rendszerek elméleteiröl és a kutatás feladatairó, trad. all. : Anti-Äquilibrium. Über die Theorien der Wirtschaftssyteme und die damit verbundenen Forschungsaufgaben, Berlin, Springer-Verlag (1975). 
Koselleck R. (1986), "Sozialgeschichte und Begriffsgeschichte ", in Schieder W. \& Sellin V. (Ed.): Sozialgeschichte in Deutschland, Goettingen, vol. I., pp. 89-109.

Kruijff G.-J. M. (2005), "Peirce's late theory of abduction: A comprehensive account ", Semiotica, 153-1/4, pp. 431-454.

Labrousse A. \& Weisz J.-D. Ed. (2000), Institutional Economics in France and Germany. German Ordoliberalism vs. the French Regulation School, Heidelberg-Berlin, Springer.

Labrousse A. (2003), Les mutations de l'économie est-allemande depuis 1990: un éclairage institutionnel et évolutionnaire, thèse de doctorat en sciences économiques, Paris, EHESS, octobre 2003.

Labrousse A. (2007) : "Le Methodenstreit: une affaire close ? ", in Alcouffe A. \& Diebold C. Ed.: La pensée économique allemande, Paris, Economica, à paraître.

Ladrière J. (1995), "Epistémologie des systèmes ", Encyclopaedia Universalis, édition CD-Rom.

Langley, P. (2004), Heuristics for scientific discovery: The legacy of Herbert Simon, in Augier M. E. \& J. G. March Ed. (2004): Models of a Man: Essays in Memory of Herbert A. Simon. Cambridge (Ma.), MIT Press, p. 463.

Lapidus A. (1996), "Introduction à une "Histoire de la Pensée Economique" qui ne verra jamais le jour ", Revue économique, 1996, vol. $47, \mathrm{n}^{\circ} 4$, pp. $867-892$.

Lapierre J. W. (1992), L'analyse des systèmes, Paris, Syros, p. 27.

Laugier S. (1999), " Pragmatisme ", in Lecourt D. Ed. (1999), pp. 750752.

Lawson T. (1997), Economics and Reality - Economics as Social Theory, London, Routledge.

Le Moigne J.-L. (1994), "Sur la capacité de la raison à discerner rationalité substantive et rationalité procédurale ", in Frydman R. Ed., Quelles hypothèses de rationalité pour la théorie économique ?, Cahiers d'économie politique,. n²4-25, Paris, l'Harmattan, pp.125159.

Lecourt D. Ed. (1999), Dictionnaire d'histoire et philosophie des sciences, Paris, PUF.

Leontief W. (1982), "A Nobel Winner's Remedy for Trial and Errors Economics ", The Christian Science Monitor, 27 août 1982, p. 23.

Lepetit B. (1996), " De l'échelle en histoire ", in Revel J. Ed. (1996), Jeux d'échelles. La micro-analyse à l'expérience, Paris, Gallimard-Le Seuil, pp. 71-94.

Lepetit B. (1996), "De l'échelle en histoire ", in Revel J. Ed. (1996), Jeux d'échelles. La micro-analyse à l'expérience, Paris, Gallimard-Le Seuil, pp. 71-94.

Lesourne J. (1994), "Chance, necessity and human willpower : a theoretical paradigm for evolutionary economics ", in Delorme R. \& Dopfer K., Ed. (1994), pp. 21-33.

50 Economie et Institutions $-n^{\circ} 8-1^{\text {er }}$ semestre 2006 
Longuet S. (2004), Des processus cognitifs à la coordination. Alfred Schütz et les Autrichiens, texte présenté au séminaire ORIS-CRIISEA du 25 mai 2004, Amiens, 60 p.

Mayr E. (2002), "Die Autonomie der Biologie ", Naturwissenschaftliche Rundschau, 55. Jahrgang, Heft 1, 2002, pp. 23-29.

Mbida J.-P. \& Pairet L. (2005), " La coopération interentreprises dans le discours des entrepreneurs : une analyse lexicométrique ", texte présenté au séminaire du CRIISEA, novembre 2005.

McCartney M. (2006), "Can a Heterodox Economist Use Crosscountry Growth Regressions?", Post-autistic economics review, issue no. 37, 28 April 2006, pp. 45-54.

Moulinié V. (1993), "La passion hiérarchique. Une ethnographie du pouvoir en usine ", Terrain, 21, pp. 129-142.

Myrdal G. (1957), Economic Theory and underdeveloped Regions, London, Duckworth \& Co.

Myrdal G. (1958), Value in Social Theory, New York, Harper.

Myrdal G. [1930]: The Political Element in the Development of Economic Theory, trad. angl., Cambridge (Mass.), Harvard University Press.

Nelson R. \& Winter N. (1982), An Evolutionary Theory of Economic Change, Cambridge (Mass.), Harvard University Press pp. 46-47.

Noiriel G. (1998) "Pour refonder l'histoire comme discipline critique ", entretien avec Roger Chartier, Le Monde des Livres, vendredi 13 mars 1998.

Nora P., Ed. (1987), Essais d'ego-histoire, Paris, Gallimard.

Orléan A. (1998), "Le poids des croyances ", Sciences Humaines, Hors-série, $\mathrm{n}^{\circ} 22$, septembre/octobre 1998, pp. 24-26.

Orléan A. (2001), "Humility in Economics ", Post-Autistic Economics Newsletter, $\mathrm{n}^{\circ} 5$, mars, article 2.

Orléan A. (2002), "Le tournant cognitif en économie ", Revue d'Économie Politique, vol.112, n5, pp. 717-738.

Orléan A. (2004), "L'économie des conventions: définitions et résultats, in Orléan A. Ed. (2004), Analyse économique des conventions, Paris, PUF, pp. 9-48.

Pareto V. [1908] : Manuel d'économie politique, trad. fr., 2e éd,. Paris, 1927, p. 260.

Pascal [1670] : Pensées, Paris, Le Livre de Poche, réed. 1972.

Peirce C. S. [1905], The Collected Papers of Charles Sanders Peirce, édité par C. Hartshorne, P. Weiss, Cambridge MA, Harvard University Press, vol. 5 (1935).

Perroux F. (1961), L'économie du XXe siècle, Paris, PUF.

Pike K.L. (1954), Language in relation to a unified theory of human behavior, The Hague, Mouton.

Rallet A. \& Torre A. (1995), Economie industrielle et économie spatiale, Paris, Economica. 
Revel J. Ed. (1996), Jeux d'échelles. La micro-analyse à l'expérience, Paris, Gallimard-Le Seuil, pp. 71-94, p. 12.

Reynaud B. Ed. (1997) : Les limites de la rationalité. Tome 2: Les figures du collectif, colloque de Cerisy, Paris, La découverte, 1997.

Saillard Y. (1995), "Globalisation, localisation et spécialisation sectorielle. Que deviennent les régulations nationales ? ", in Boyer R. \& Saillard Y. (1995), pp. 285-292.

Sapir J. (1999), "Le capitalisme au regard de l'Autre ", in Chavance et al. Ed. (1999), Capitalisme et socialisme en perspective. Evolution et transformation des systèmes économiques, Paris, La Découverte, pp. 185-216.

Sapir J. (2000), Les trous noirs de la science économique, Paris, Albin Michel.

Schmoller G. (1869), "Die neueren Ansichten über Bevölkerungsund Moralstatistik ", in: Schmoller G. (1888): Zur Literaturgeschichte der Staats- und Sozialwissenschaten, Leipzig, Duncker \& Humblot, pp. 172-203

Schmoller G. (1911), "Volkswirtschaft, Volkswirtschaftslehre und methode ", Handwörterbuch der Staatswissenschaften, réédité dans Historisch-ethische Nationalökonomie als Kulturwissenschaft, (1998), Marburg Metropolis Verlag, édité par H.H. Nau.

Simon H.A. (1997), Models of Bounded Rationality - Empirically Grounded Economic Reason, vol. 3, MIT Press, pp. 368-369.

Simon H.A. (1997), Models of Bounded Rationality: Empirically Grounded Economic Reason, vol. 3, MIT Press, pp. 368-369.

Simon H.A. [1969]: Sciences des systèmes, sciences de l'artificiel, trad. fr., Paris, Dunod (1991).

Thelen K. \& Steinmo S. (1992), "Historical Institutionalism in Comparative Politics ", in Steinmo S., Thelen K. \& Longstreth P. Ed. (1992), Structuring Politics, Cambridge, Cambridge University Press, pp. 1-32.

Théret B. (1997), "Méthodologie des comparaisons internationales, approches de l'effet sociétal et de la régulation : fondements pour une lecture structuraliste des systèmes nationaux de protection sociale ", L'Année de la Régulation, 1, Paris, La Découverte, pp. 163-228.

Théret B. (2000), "Institutions et institutionnalismes : vers une convergence des conceptions de l'institution ? " in Tallard M., Théret B. \& Uri D. (2000), Innovations institutionnelles et territoires, Paris, L'Harmattan, 2000, pp. 25-68.

Théret B. (2001), "Saisir les faits économiques: une lecture structuraliste génétique de la méthode de Commons", Cahier d'économie politique, $\mathrm{n}^{\circ}$ 40-41, pp. 79-137.

Veblen T. (1898), "Why is Economics Not an Evolutionary science ?", The Quarterly Journal of Economics, vol. 12, No. 4., pp. 373-397.

52 Economie et Institutions $-n^{\circ} 8-1^{\text {er }}$ semestre 2006 
Veblen T. [1899-1900], "The Preconceptions of Economic Science ", I, II, III, The Quaterly Journal of Economics, jan. 1899, jul. 1899, feb. 1900, reed. in : The Collected Works of Thorstein Veblen, vol. VIII, The Place of Science in Modern Civilisation and Other Essays, London, Routledge-Thoemmes Press, (1994).

Veblen T. [1915] : Imperial Germany and the Industrial Revolution, The Collected Work of Thorstein Veblen, vol. 4, London, Routledge (1994).

Weber F. (1996), "Métier d'historien, métier d'ethnographe ", Cahiers Marc Bloch, 4/1996, pp. 6-24.

Weber M. (1919), Wissenschaft als Beruf. München-Leipzig, Duncker $\&$ Humblot (Geistige Arbeit als Beruf. Vorträge vor dem Freistudentischen Bund).

Weber M. [1904-1917] (1959), Essais sur la théorie de la science, trad. fr. et intr. de Julien Freund, Paris, Plon.

Weber M. [1905] (2003), L'éthique protestante et l'esprit du capitalisme, suivi d'autres essais, trad. Fr. de J.-P. Grossein, Paris, Gallimard.

Weber M. (1922), Wirtschaft und Gesellschaft, Tübingen, Mohr. 